\title{
Socioeconomic position and body composition in childhood in high- and middle-income countries: a systematic review and narrative synthesis
}

\author{
Charis Bridger Staatz $\mathbb{D}^{1 凶}$, Yvonne Kelly $\mathbb{D}^{2}{ }^{2}$, Rebecca E. Lacey $\mathbb{D}^{2}$, Joanna M. Blodgett $\mathbb{D}^{3}$, Anitha George (D) ${ }^{2}$, Megan Arnot $\mathbb{D}^{4}$, \\ Emma Walker ${ }^{2}$ and Rebecca Hardy (D) $^{1}$ \\ (c) The Author(s) 2021
}

\begin{abstract}
BACKGROUND: The relation between socioeconomic position (SEP) and obesity measured by body mass index (BMI), a measure of weight for height, has been extensively reviewed in children, showing consistent associations between disadvantaged SEP and higher BMI in high-income countries (HICs) and lower BMI in middle-income countries (MICs). Fat mass (FM), a more accurate measure of adiposity, and fat-free mass (FFM) are not captured by BMI, but have been shown to track from childhood to adulthood, and be important for cardiovascular health and functional outcomes in later life. It is not clear whether body composition is associated with SEP. We systematically reviewed the association between SEP and body composition in childhood.

METHODS: A systematic review was carried out following PRISMA guidelines. The protocol was pre-registered with PROSPERO (CRD42019119937). Original studies in the English language, which examined the association between SEP and body composition in childhood, were included. An electronic search of three databases was conducted. Two independent reviewers carried out screening, data extraction and quality assessment. Due to heterogeneity in results, a narrative synthesis was conducted. Heterogeneity in findings according to SEP, sex, body composition measure and country income level was investigated. RESULTS: 50 papers were included, the majority from HICs. No papers were from low-income countries. Disadvantage in childhood was associated with greater FM and lower FFM in HICs, but with lower FM and lower FFM in MICs. When measures of FFM indexed to height were used there was no evidence of associations with SEP. In HICs, more studies reported associations between disadvantaged SEP and higher FM among girls comparative to boys.

CONCLUSIONS: Inequalities in FM are evident in HICs and, in the opposite direction, in MICs and follow similar trends to inequalities for BMI. Inequalities in height are likely important in understanding inequalities in FFM.
\end{abstract}

International Journal of Obesity (2021) 45:2316-2334; https://doi.org/10.1038/s41366-021-00899-y

Childhood obesity is a globally recognised public health challenge and is a major determinant of obesity in adulthood [1]. Previous systematic reviews investigating the link between socioeconomic position (SEP) and obesity measured through body mass index (BMI) in childhood have predominantly found disadvantaged SEP to be associated with higher levels of obesity in high-income countries [2, 3], especially when SEP was measured by parental education [2]. In low- and middleincome countries, disadvantaged SEP is associated with lower levels of obesity [4]. Compared with studies in adults where sex differences have been observed, with women typically demonstrating greater evidence of inequalities, little evidence of stronger associations in girls compared to boys in high-income countries has been found [2].
The majority of evidence on inequalities in overweight and obesity in children comes from studies using BMl, a measure of weight for height which does not distinguish fat mass (FM) from fat-free mass (FFM) and therefore may under or overestimate adiposity. Measures of body composition can provide information about the location of FM and estimates of the proportion of FM to FFM. FFM includes bone mass and lean mass (LM) and is most frequently measured by bioelectrical impedance analysis (BIA). LM is a measure that excludes bone mass and is most frequently measured through dual x-ray absorptiometry (DXA) [5]. In adults, a higher proportion of fat-to-lean mass is associated with a higher risk of cardiovascular disease [6]. Both total and proportion of fat mass have been associated with cardiovascular and metabolic disease, with higher central adiposity and android-to-gynoid fat

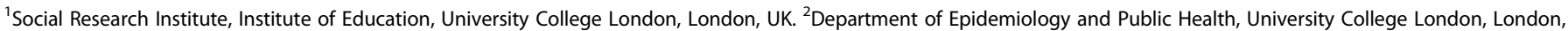

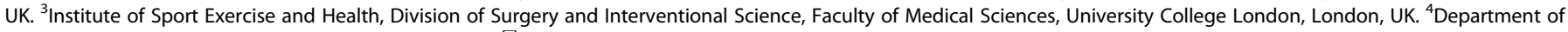

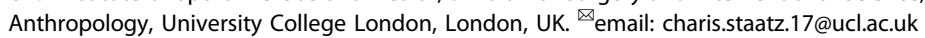

Received: 5 December 2020 Revised: 24 May 2021 Accepted: 30 June 2021

Published online: 27 July 2021 
mass ratio implicated in increased risk [7-10]. In addition, LM plays a role in development of insulin sensitivity, with muscle tissue being a site of glucose uptake, therefore having the potential to reduce and delay the onset of metabolic disorders [11, 12].

Studies using serial data in children have shown secular changes in body composition, with an increasing trend for FM index (FMI) from 1960 to 1999 in the US [13]. In the UK, decline in muscle fitness, as measured by strength, power and strengthendurance, has been observed among children, when adjusted for height and weight, between 1998 and 2014 [14]. It is possible that such secular changes in body composition are accompanied by increases in socioeconomic inequality in body composition, as has been observed for BMI [15], where increases in inequalities are particularly evident across childhood [16]. Additionally, secular changes in muscle and fat acquisition in childhood may subsequently result in detrimental secular changes in adult body composition. As people age, BMI increases are more likely to reflect fat acquisition than muscle [17] and tracking of body composition from childhood to adulthood has been demonstrated $[18,19]$.

We therefore carried out a systematic review to assess the association between SEP and measures of body composition (in particular FM, FFM, and the location of FM) in children (up to and including 18 years) from general population samples. Additional aims were to assess secular changes in socioeconomic inequalities in body composition and explore heterogeneity by sex, SEP measure, body composition measure and income level of country of study.

\section{METHODS}

The protocol for this review, which is the second part of a larger systematic review investigating socioeconomic inequalities in adults [20], was registered with the PROSPERO database (CRD42019119937). The review has been carried out according to Preferred Reporting Items for Systematic Reviews and MetaAnalyses (PRISMA) checklist (Supplementary File 1). Further details of the methods can be found in the published protocol [21].

\section{Eligibility}

Peer reviewed papers written in the English language were included in this review if they reported an association between SEP and body composition in children (under 18 years of age) using data from an observational study including a sample from the general population. Associations between any recognised indicator of SEP (e.g., income, education, overcrowding, area-level deprivation) and a measure of body composition, measured at the same, or later, time point to SEP were included. As studies in this review are in children, measures of SEP, such as occupation and education, relate to parents, other markers such as overcrowding reflect the home in which the child lives, and some markers of education are based on the type of school attended. Body composition (i.e., measured using BIA or DXA) was defined as any measurement related to total FM and FFM, location of FM and FFM or any proportion or ratio of measures of FM and FFM.

\section{Search strategy}

CBS conducted an electronic search of three databases (MEDLINE and Embase Classic + Embase using OvidSP as the interface, SPORTDiscus using EBSCO as the interface) from the earliest entry up until the 30th of January 2019. The search terms used are shown in Table 1 and include adult as well as childhood samples. The results of the search were de-duplicated and stored in the reference manager, Endnote. This database was exported to Rayyan Qatar Computing Research Institute (QCRI) [22] to conduct screening. CBS, AG, and JB conducted title and abstract screening for eligibility, and subsequent full text screening of eligible papers for inclusion in the review. Additionally, the reference list of eligible full texts were screened and searches of publications from key studies with relevant data were used to identify further papers.

\section{Extraction and quality assessment}

Relevant information was double extracted using a data extraction form by CBS, AG, JB, MA, and EW. Data extracted included citation details (author, title, publication year, publication type), study details (cohort or sample description, study design, country, participant numbers), participant details (birth year or age of participants, sex of participants), exposure and outcome details (type of SEP and body composition variables presented, age at which variables were recorded, how the variables were ascertained and measured) and statistical methods and information on adjustment for potential confounders and mediators. All available statistics relating to the association under study were extracted, along with statements of direction in text where statistics were not presented.

Assessment of study quality was carried out by CBS, AG, JB, MA, and EW, using an amended version of the Newcastle-Ottawa Quality Assessment scale [23]. Quality assessment was not used to exclude papers from the review, but to inform on the variability of quality across the papers and potential bias arising. The quality assessment form was amended after the protocol was published to account for the variability in statistical reporting and the large number of cross-sectional studies identified (questions 3bi, 3bii and 4 - Supplementary File 2). Google Forms was used to aid extraction and WebPlotDigitizer [24] was used to extract data only presented in graphs.

Two reviewers (CBS and either AG, JB, MA or EW) worked independently to complete screening, quality assessment and data extraction. Any disagreements were resolved through discussion.

\section{Synthesis}

A meta-analysis was not possible due to the considerable variability in analytic methods used and presentation of results. As such, it was not possible to assess the degree of publication bias across studies through use of a funnel plot. Instead, a narrative synthesis was conducted, guided by the Economic and Social Research Council Methods Programme guidelines [25], with a focus on identifying and exploring sources of heterogeneity. The current review reports results only for associations in childhood due to the large number of papers included. The results for adulthood are reported elsewhere [20].

Multiple relevant associations were frequently presented in a single paper. The individual association, as opposed to the paper, were thus considered the unit of analysis, similar to methods adopted by McLaren [26], and Ball and Crawford [27]. This will have resulted in greater contribution of results from a single paper where multiple associations were reported.

Each association reported was categorised as either a positive association (those reporting greater socioeconomic advantage associated with higher body composition measure), negative association (those reporting greater socioeconomic advantage associated with lower body composition measures), non-linear association or no association. We removed the non-linear group from the summary tables, similar to the approach of McLaren [26], as only one association fell into this category. Associations were assigned to groups based on the effect estimates and 95\% confidence intervals. Where estimates were not reported, assignment was based on trends identified in descriptive data or statements of direction reported in text alongside $P$ values. Use of $P$ values on their own only occurred if they indicated a non-significant relationship in absence of information on the direction of association.

As outlined in the protocol, heterogeneity in associations was explored according to body composition measure (FM, FFM, ratio and distribution), SEP measure and sex. Results from analyses 
Table 1. Search terms.

\section{Search terms}

Database

Medline

Embase + Embase Classic

\section{SPORTDiscuss}

Free Text Search Terms Body composition

Body composition Measures

\section{MeSH Terms}

Body Composition - exp Body Composition/; Adipose Tissue/; exp Body Fat Distribution/; Obesity/or obesity, abdominal/.

Body Composition Measures - Electric Impedance/; Magnetic Resonance Imaging/; Tomography, X-Ray Computed/; Densitometry/; Whole-Body Counting/; Plethysmography/.

Socioeconomic Position - socioeconomic factors/ or poverty/ or poverty areas/ or social class/; Educational status/ or income/ or occupations/ or social conditions/.

Body Composition - Body composition/ or body distribution/ or body fat/ or body fat distribution/; Obesity/; lean body weight/; Fat mass/.

Body Composition Measures - Impedance/; nuclear magnetic resonance imaging/; computer assisted tomography/; densitometry/; whole-body counting/; Total body water/; plethysmography/.

Socioeconomic Position - socioeconomics/ or educational status/ or income group/ or poverty/; income/ or occupation/ or household income/; social status/ or social background/ or social class/; education/;

Body Composition - ((DE "BODY composition" OR DE "HUMAN body composition") OR (DE "OBESITY")) OR (DE "ADIPOSE tissues")

Body Composition Measures - ((((DE "BIOELECTRIC impedance") OR (DE "COMPUTED tomography")) OR (DE "MAGNETIC resonance imaging")) OR (DE "BONE densitometry")) OR (DE "PLETHYSMOGRAPHY")

Socioeconomic Position - ((DE "EDUCATION") OR (DE "EDUCATIONAL attainment")) OR (DE "HEALTH \& income")

1. Body Composition MeSH Terms

2. (Body adj3 (composition or distribution))

3. ((fat or adipos*) adj3 (composition or distribution or mass or index or kg or total))

4. ((muscl* or lean) adj3 (composition or distribution or mass or index or kg or total))

5. ((fat-free) adj3 (mass or kg or total))

6. ((android or gynoid or visceral or appendicular or abdominal or intra-abdominal) adj3 (fat or lean or muscle or mass or adipos*))

\section{1 OR 2 OR 3 OR 4 OR 5 OR 6}

\section{Body Composition Measures MeSH Terms}

9. ((impedance) adj3 (bioelectrical or foot-to-foot or hand-to-foot or analy?is))

10. (bioimpedance or body fat analy?er or body composition analy?er or tanita)

11. (dual x-ray absorptiometry or DEXA or DXA or dual-energy X-ray absorptiometry)

12. (magnetic resonance imaging or MRI)

13. (Computed tomography or CT or CAT scan)

14. (densitometry)

15. ((neuron activation or total body counting or whole-body counting))

16. (total body water)

17. (air-displacement plethysmography)

18. 8 OR 9 OR 10 OR 11 OR 12 OR 13 OR 14 OR 15 OR 16 OR 17

19. 7 AND 18

20. Socioeconomic Position MeSH terms

21. (social class or social status or social position or socio-economic or socioeconomic or social circumstance ${ }^{*}$ )

22. (sociodemo*)

23. Occupation*

24. Educat*

25. (income* or manual or class)

26. (depriv* ${ }^{*}$ or poverty or overcrowding)

27. 20 OR 21 Or 22 OR 23 OR 24 OR 25 OR 26

28. 19 AND 27

29. Limit to English Language (and Human in OvidSP)

MeSH terms are main heading descriptor terms available in each database and are determined by the indexing method adopted by each database. Free text search terms were entered into all databases, along with the results of the database specific MeSH terms. 
using boys and girls combined were the primary results selected for summary. Where results were only presented for girls and boys separately, both associations were included in the summary results. It was not possible to investigate differences in body composition by birth year as outlined in the protocol, due to lack of information provided on birth year across studies. On extraction, it became clear that country income level should also be considered a source of heterogeneity. Studies were thus categorised into those in high-income countries (HIC), upper middle and lower middle-income countries, according to the World Bank classification in 2019 [28]. Those papers from "upper middle" and "lower middle" income countries will all be referred to as "middle-income countries" (MIC). On the suggestion of a reviewer, we also investigated impact of paper quality on the findings.

\section{RESULTS}

In total, 7145 papers were identified from the database searches for studies in both children and adults, with 5725 once duplicates were removed. Title and abstract screening resulted in 513 papers, with 92 papers remaining following full text screening. Searching the reference lists for additional papers returned three, bringing the total included papers to 95 . Of those, 48 investigated associations between SEP and childhood body composition. A search of papers from key studies resulted in two further papers in children being identified, bringing the total number of included papers to 50 [18, 29-77]. The selection process, as outlined in the PRISMA flow chart, is shown in Fig. 1. Descriptive results for the included papers are shown in Table 2. The majority of papers were rated as medium quality in the adapted Newcastle-Ottawa assessment and eight studies were rated as high quality $\left(\geq 7^{*}\right)$ and ten as low quality $\left(\leq 3^{*}\right)$. Those rated as high quality all presented full statistical results, including effects estimates and confidence intervals, whilst those rated as low quality typically had statistical reporting deemed inappropriate or incomplete. Only one paper presented $P$ values alone to report a non-significant result without provision of effect estimates, descriptive data or statement of direction in text.

\section{Characteristics of included studies}

There were 38 distinct samples studied across the 50 papers. The Avon Longitudinal Study of Parents and Children (ALSPAC) and a sample from Merida, Mexico were the most commonly included studies, appearing in four and three separate papers, respectively. The majority of papers were conducted in population samples from high-income countries $(n=36,72 \%)$ with the remaining papers from MICs $(n=14)$. The UK and the US contributed the most papers ( $n=10$ in the UK, $n=8$ in the US), with 7 unique studies in both. Sample size across the papers ranged from 74 to 14,314, with a median sample size between 485 and 502.

There was substantial variation in body composition measures used, the definitions of which are outlined in Supplementary Table 1. In this review, we use fat-free measures as a general term referring to any measure of body composition not including fat mass. These measures include FFM, which represents total mass with fat mass excluded, and lean body mass (LBM), a measure of FFM plus essential fats, which are most commonly measured by BIA [78]. Other measures are dry lean mass (DLM), which is LBM without body water, and lean mass (LM), a measure of FFM that excludes bone and is more similar to what is colloquially understood as muscle [79] and is most often measured by DXA. In this review, appendicular skeletal muscle is considered a total body fat-free measure, as muscle mass in the limbs captures $75 \%$ of total skeletal muscle mass (SMM), and therefore is a good indicator of total body muscle $[79,80]$.

Fat measures were considerably more frequently reported than FFM measures (in 46 papers compared to 22), with percentage body fat being the most commonly analysed (29 papers). Among papers that investigate fat-free measures, LM was the most frequently used (8 papers). The majority of papers used either dual x-ray absorptiometry (DXA) $(n=25)$, or bioelectrical impedance (BIA) analysis $(n=22)$ to measure body composition, with two papers using both methods. Five studies used air-displacement plethysmograph with one of these also using BIA. One other paper used deuterium dilution in combination with DXA and plethysmography. The SEP variable most frequently reported was parental education $(n=25)$.

A similar number of studies were conducted in children aged, or with a mean age, between four and ten $(n=21)$ as were conducted in those children and adolescents over the age of ten $(n=24)$. One study was conducted in new-borns and was the only study to be conducted in children under the age of four.

\section{Childhood SEP and total fat mass measures}

Table 3 provides a summary of the patterns of association reported for total FM measures. There were 124 associations tested across 46 papers. Negative associations, where more advantage SEP was associated with lower fat, were reported most often, in $42 \%$ (52 association across 31 papers) of the 124 associations. The remaining associations were split between positive associations (27\%, 33 associations from 7 papers), where more advantaged SEP was associated with greater fat, and no association (31\%, 39 association from 19 papers).

In HICs, associations were predominantly negative (66\%, 46 associations from 28 papers) with greater socioeconomic advantage being associated with less fat. The remaining associations in HICs all showed no overall pattern of association (24 association from 13 papers). In MICs, the majority of associations were positive (61\%) with greater socioeconomic advantage associated with higher levels of fat. Only 11\% (6 associations from 3 papers) reported negative associations, with the remaining associations (28\%, 15 associations from 6 papers) reporting no overall pattern.

The total body fat measure most frequently reported was FM\%, being used 60 times (across 29 papers), followed by FM used 38 times (18 papers), and FMI used 26 times (13 papers). In HICs, using $\mathrm{FM}$ or $\mathrm{FM} \%$ yielded a slightly greater number of negative associations, where greater advantage is related to lower levels of fat, $(67 \%$ and $68 \%$, respectively) compared to $\mathrm{FMI}(60 \%)$. In MICs, FM and FMI presented almost exclusively positive associations, where greater advantage is related to higher levels of fat $(79 \%$ and $82 \%$, respectively). FM\% exhibited more mixed results with $45 \%$ finding positive associations, $17 \%$ finding negative and the remaining 38\% finding no association.

Parental education was the most commonly used SEP measure across the papers, used in 53 associations across 24 papers. In MICs, composite measures of SEP were the most frequently recorded SEP measure, used in 22 associations across nine papers. Among $\mathrm{HICs}$, negative associations were reported in the majority of associations ( $\geq 60 \%$ ) for all SEP measures, with the exception of parental or household income, used in six papers, where no association was most frequently reported ( 6 association from four papers, out of 10 associations). In MICs, parental education, used in seven papers, yielded a higher number of positive associations (15 association from five papers, out of 18 associations, 83\%) compared with composite SEP measures (14 associations from four papers, out of 22 associations, 64\%).

Sex-specific analysis was presented in 15 papers in HICs and eight papers in MICs, with 44 and 37 associations reported, respectively (Fig. 2). Negative associations were more frequently reported among girls (83\%) compared with boys (43\%) in HICs. In MICs positive associations were somewhat more commonly reported in girls (78\%) compared to boys $(63 \%)$. Boys in both HICs and MICs were more likely to show no association between SEP and fat measures (HIC: $57 \%$; MIC: $26 \%$ ) compared to girls in either (HIC: 19\%; MIC: 17\%). 


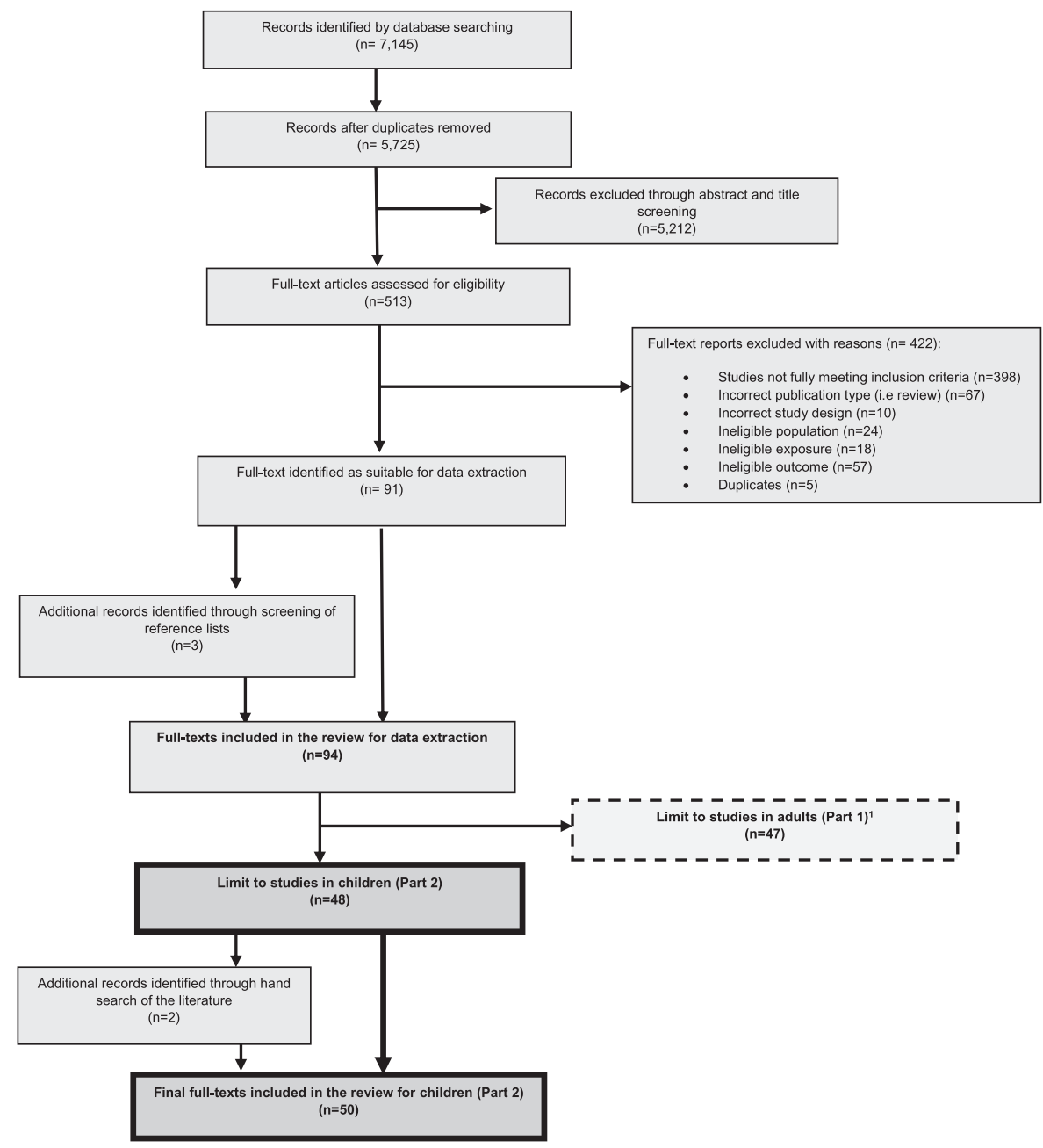

Fig. 1 Study selection process outlined with PRISMA flow chart. Because reasons for exclusion are not mutually exclusive, numbers given for reasons for exclusion during full text-screening stage equal more than the total excluded at this stage $(n=422)$. Number of studies identified in adults and children is greater than total full texts included in the review, as one paper covered childhood and adulthood and was used in both reviews. ${ }^{1}$ Studies in adults are reported on elsewhere.

When considering results in high quality papers only (rated $\geq 7^{*}$ in the quality assessment), findings were similar to the full analysis. In HICs nine associations out of 13 (69\%), reported across six papers, showed greater socioeconomic advantage associated with higher levels of fat. In MICs, three out of five associations (60\%), reported in two papers, found greater advantage associated with higher FM. In low-quality papers $\left(\leq 3^{*}\right)$ eight out of 13 associations (62\%) from five papers reported negative associations similar to that in high quality papers. However, in MICs, the low-quality papers, contrasting with results in the full analysis, showed predominantly negative associations. However, the findings were from only two papers both conducted in the same population sample.

\section{Childhood SEP and total fat-free mass measures}

Table 4 provides a summary of the patterns of association for total body FFM measure. There were 69 associations tested across 22 papers. Approximately half (33 associations in 13 papers) found positive associations (48\%), with greater socioeconomic advantage being related to greater FFM. Only 12\% demonstrated negative associations, with the remaining $41 \%$ reporting no association. Positive associations were reported more frequently in HICs (55\%) compared to MICs (43\%), whilst negative associations were only reported in MICs (20\%).
Raw fat-free measures, used in 14 papers, show positive associations in $59 \%$ of analyses (20 associations coming from nine papers, out of 34 associations), more frequently than both percentage measures $(50 \%, 11$ associations coming from six papers, out of 22 associations across eight papers) and considerably more often than indexed measures (15\%, two associations coming from two papers, out of 13 associations across five papers). Measures that include bone in their assessment show positive associations slightly more often (54\%) than those which exclude bone (42\%).

Parental education was the most frequently investigated SEP measure, used in 24 associations. Composite measures of SEP were also frequently used $(n=18)$, with all except one such association tested in MICs. Parental occupational social class and measures of area-level SEP were used in eight and nine associations respectively, although for area-level SEP, eight were all tested in the same paper. Parental income was used three times in two papers. In MICs there was a slightly higher number of positive associations reported when using composite measures of SEP (53\%) compared to education (40\%). In HICs, approximately one-third of associations with both education and occupational social class were observed to be positive. In the small number of analyses including area-level SEP and income in $\mathrm{HICs}$, only positive associations were seen. 


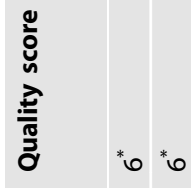

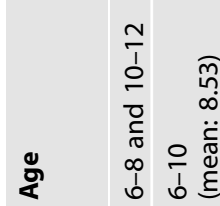

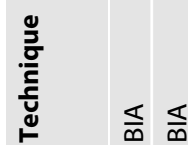

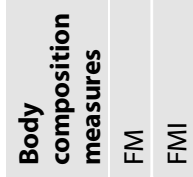

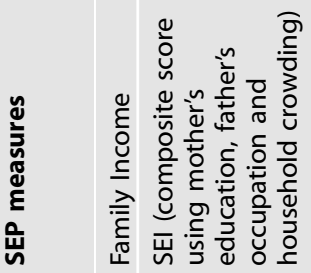

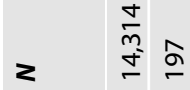

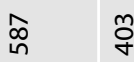

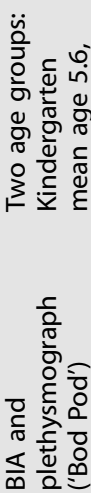

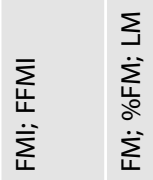

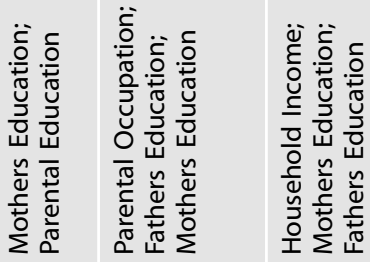

ญั

$\sum_{\frac{1}{4}}^{\sum_{0}^{0}}$

$\stackrel{n}{2}$

$\sum$
*

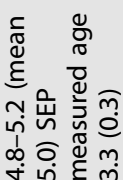

in $\mathrm{Em}$

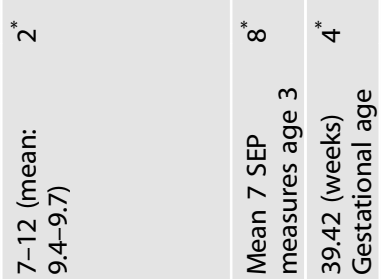

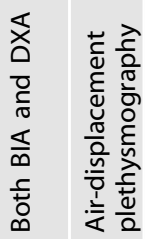

亮这

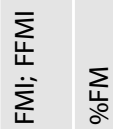

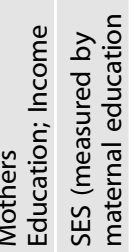

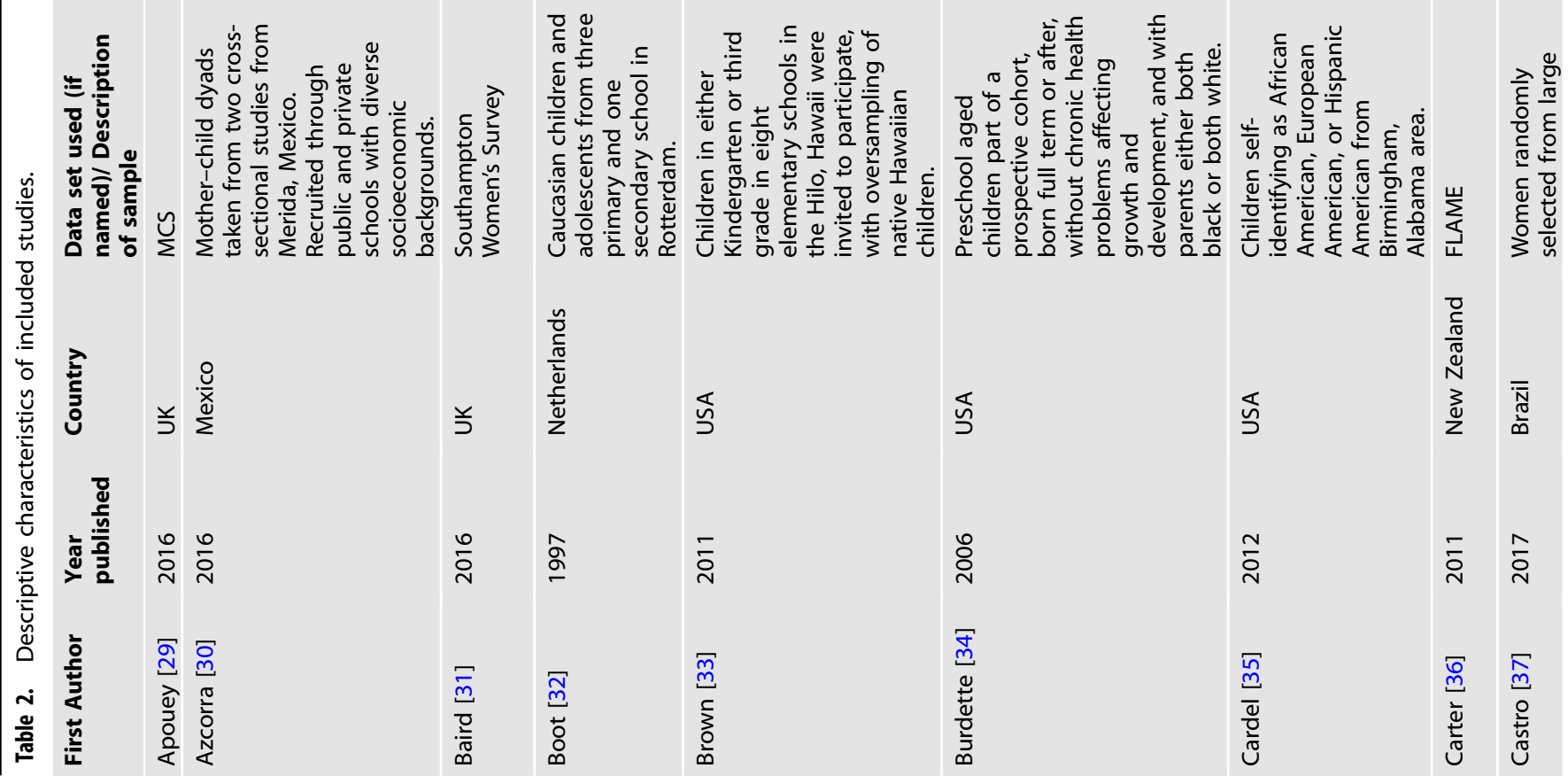




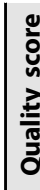

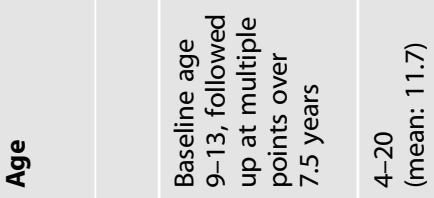

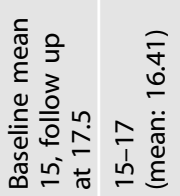

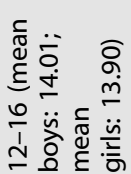

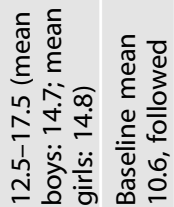

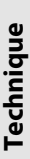

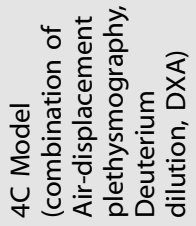

ষ্ড

$\overleftarrow{\mathrm{c}}$

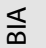

ড়

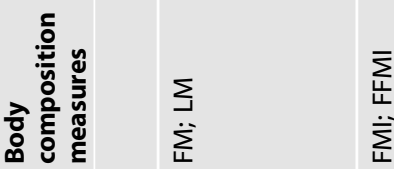

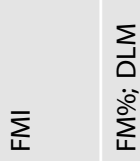

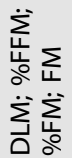

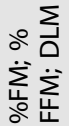

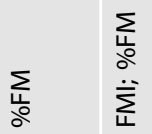

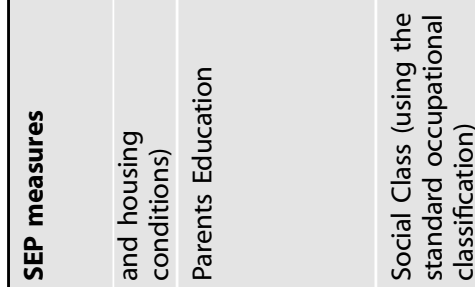

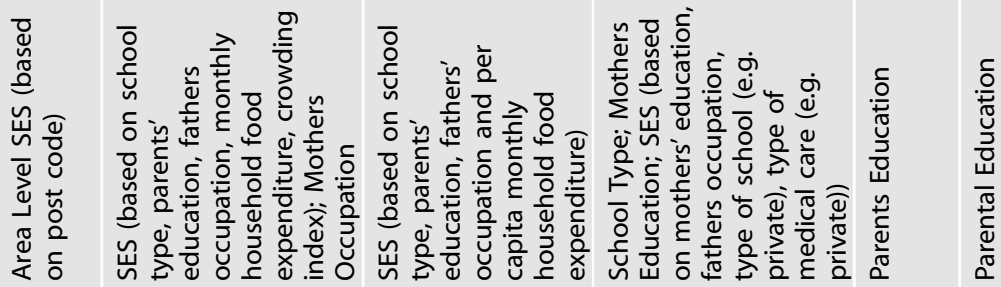

$2 \quad$

임

$\stackrel{\bar{m}}{\sim}$

ז

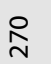

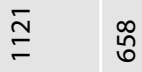

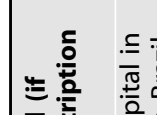

产

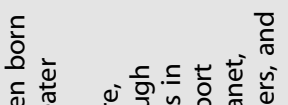

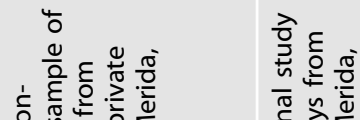

ร

亏 $\quad \frac{8}{\bar{x}}$

$\frac{\stackrel{0}{x}}{\sum^{0}}$

$\frac{\stackrel{0}{x}}{\sum^{2}}$

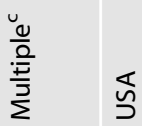



ঃั

ֻ

in

$\bar{i}$

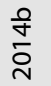

$\overline{\text { ก }}$

$\infty$
$\stackrel{\infty}{\Xi}$
0
$\bar{\Phi}$
ป

啹

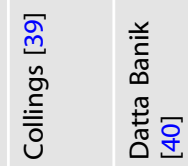

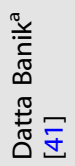

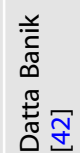

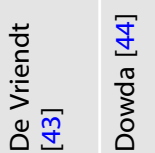




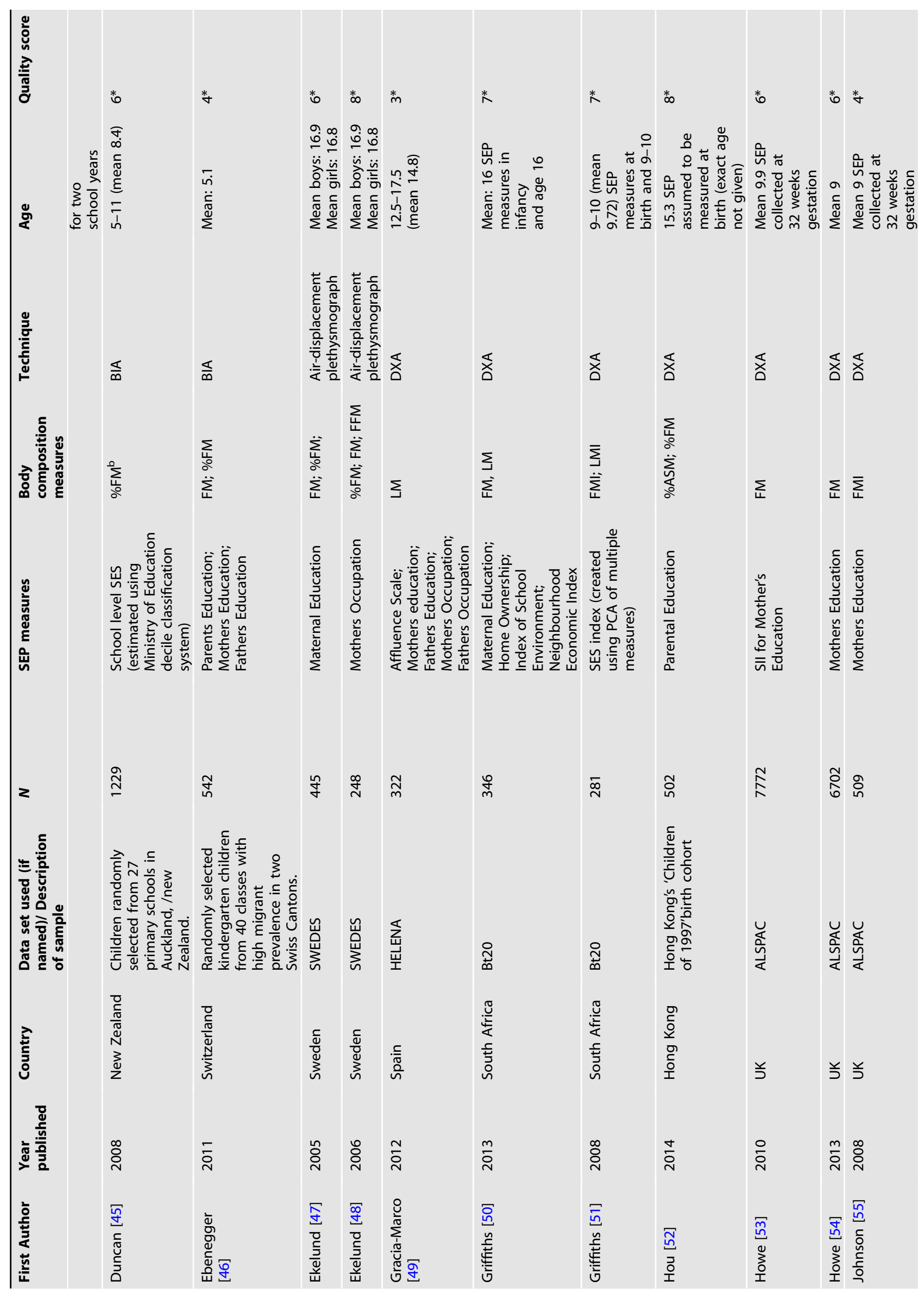


㐫

$\stackrel{\frac{5}{\infty}}{\bar{m}}$

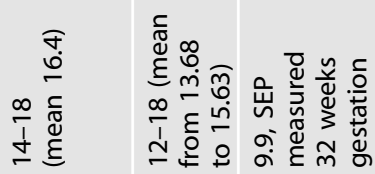

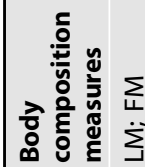

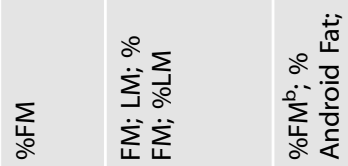

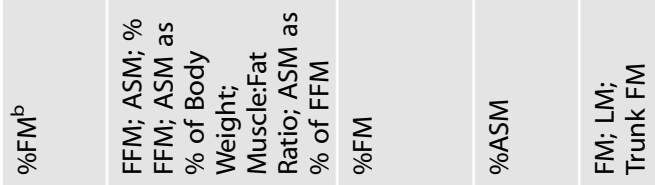
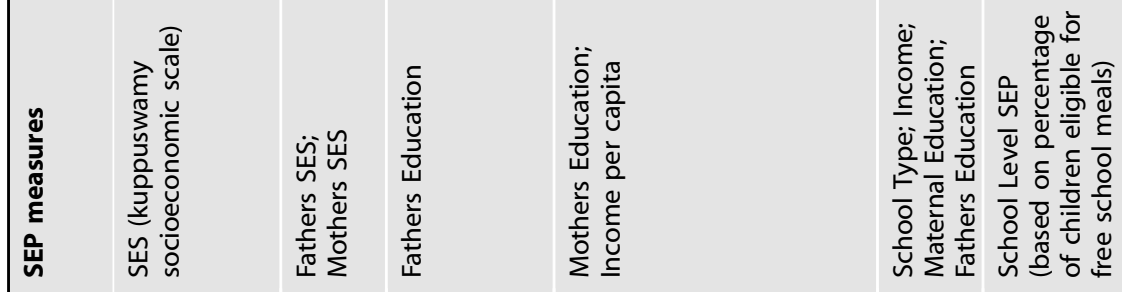

產高

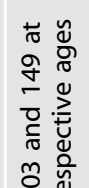

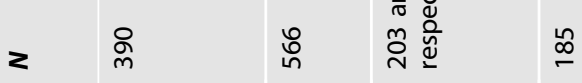

吕

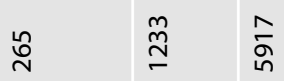

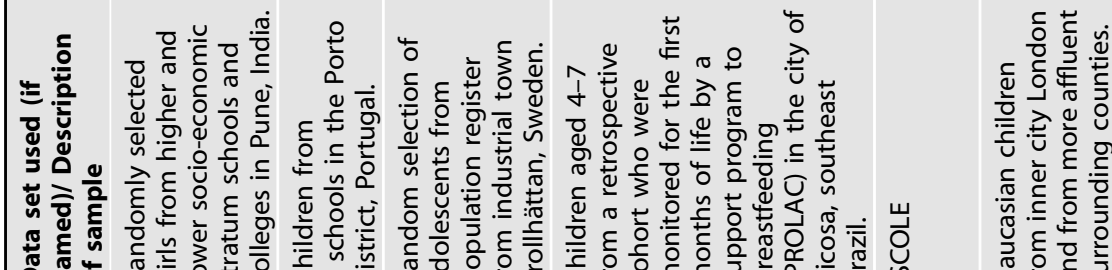

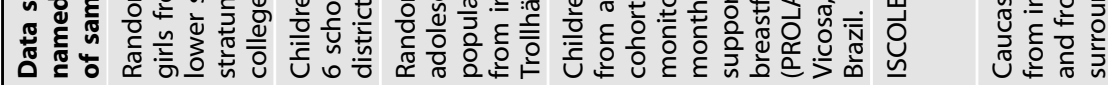

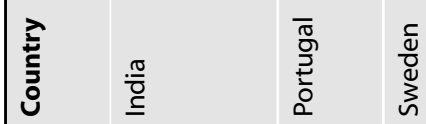

$\overline{\overline{\mathrm{D}}} \overline{\mathrm{D}}$

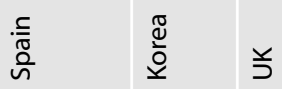

离

๙ั)

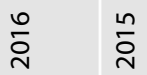

సे

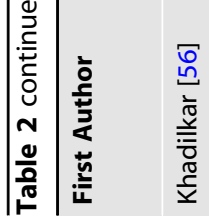

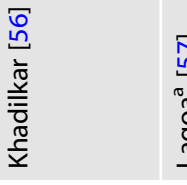

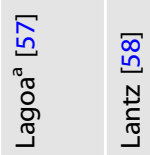

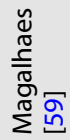

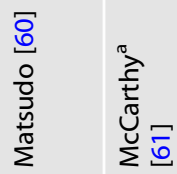

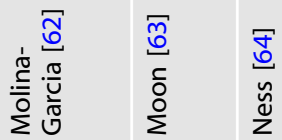




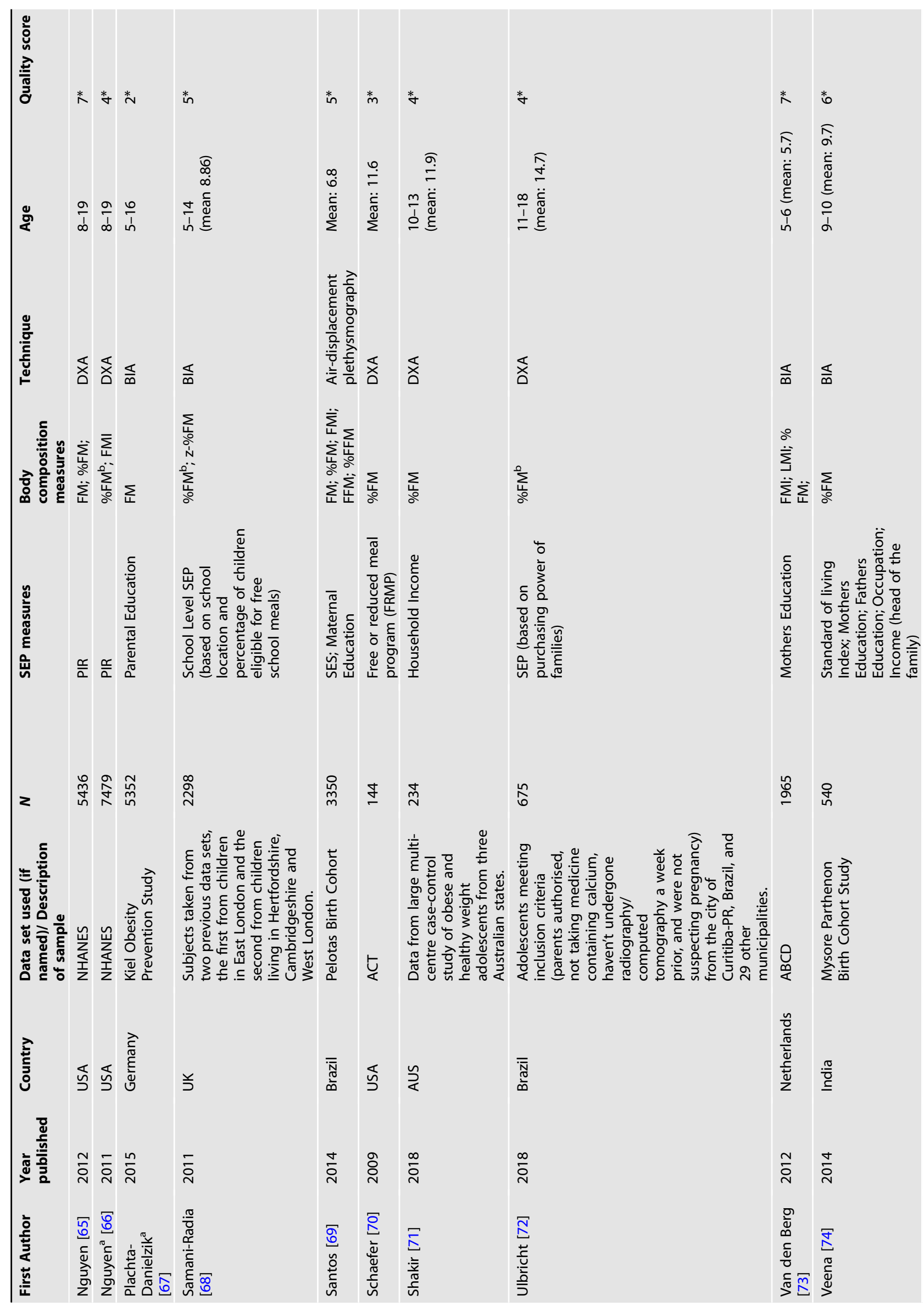




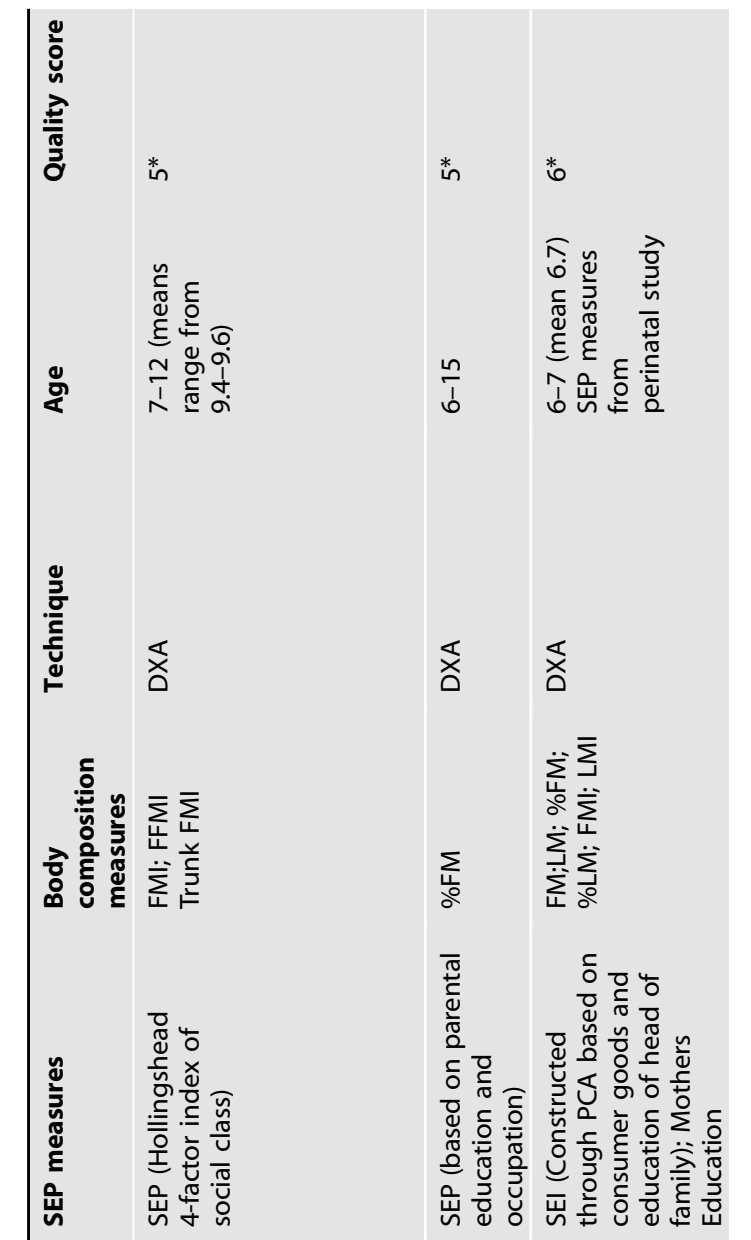

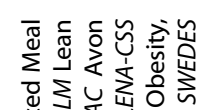

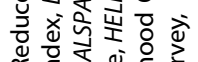

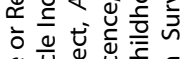

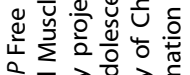

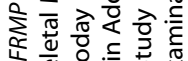

은

要

일

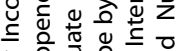

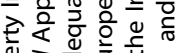

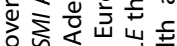

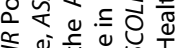

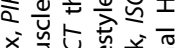

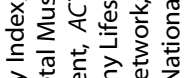

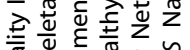

要

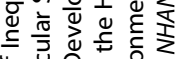

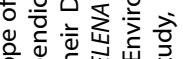

은 응

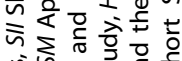

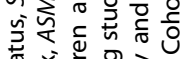

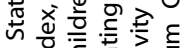

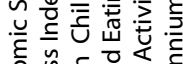

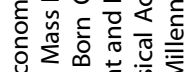

ญ्ष

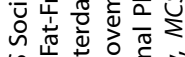

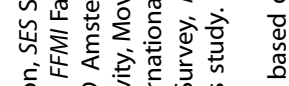
.

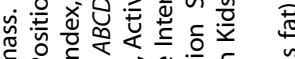

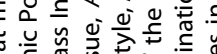

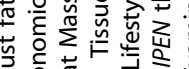

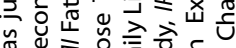

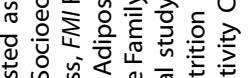

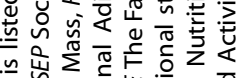

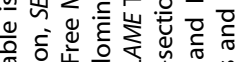

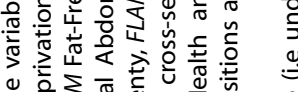

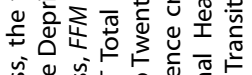

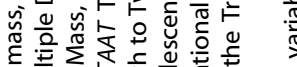

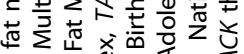

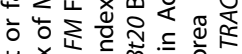
荡
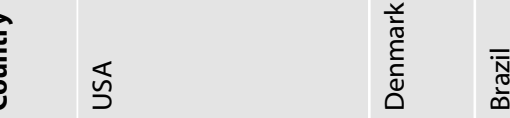

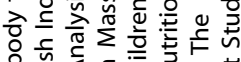

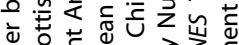
辛讪

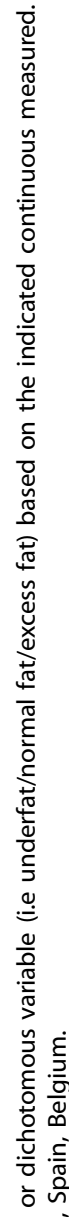

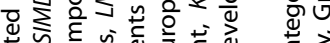

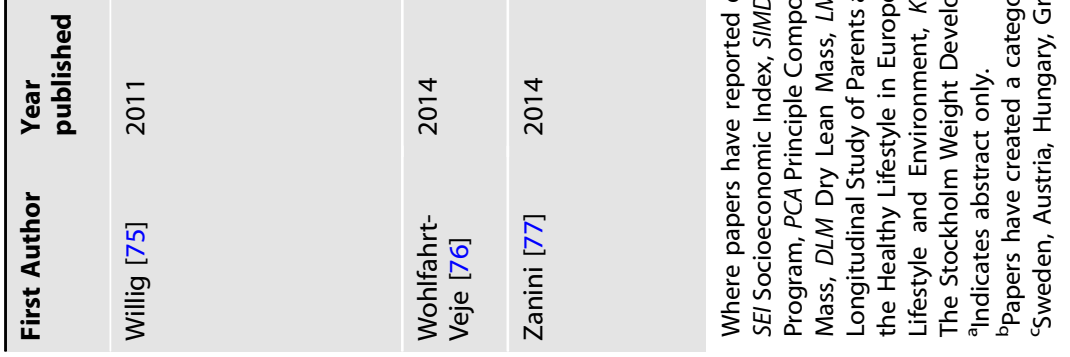




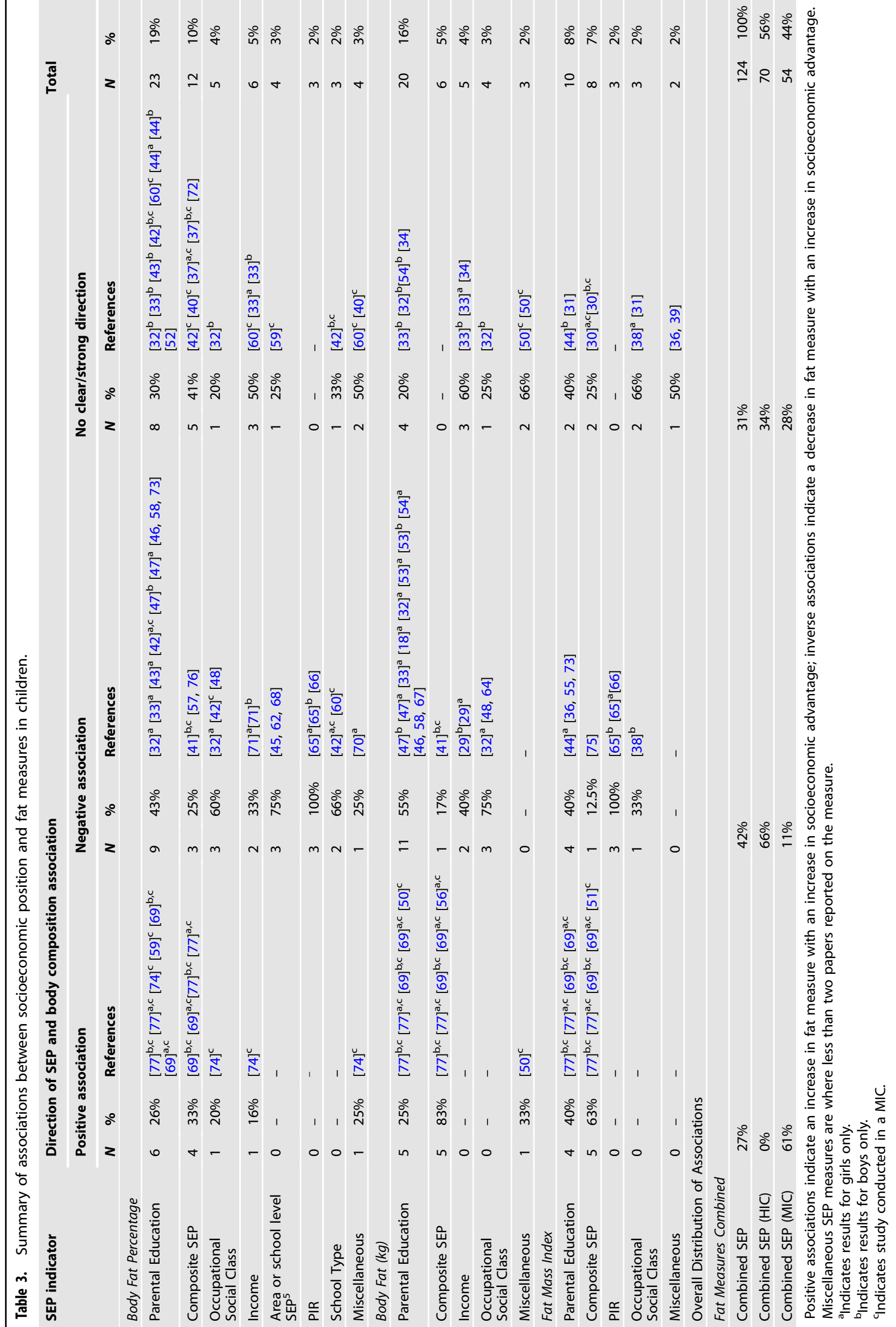




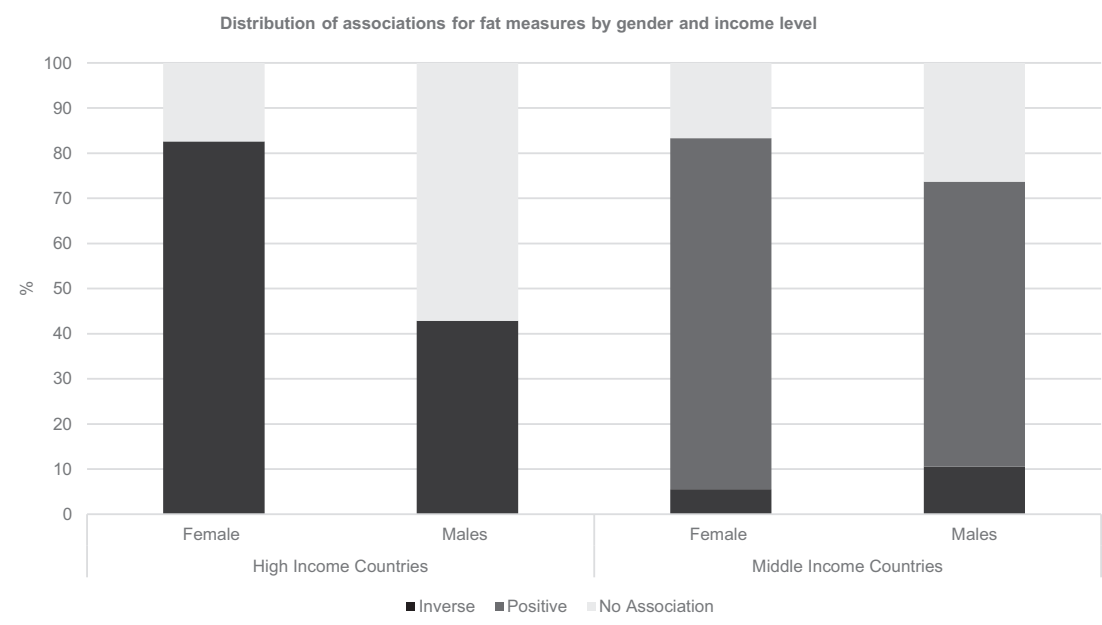

Fig. 2 Distribution of associations for fat measures by gender and income level. Girls in HICs $(N=23): 83 \%$ negative associations, $0 \%$ positive associations, $0 \%$ non-linear associations, $17 \%$ show no association; Boys in HICs $(N=21): 43 \%$ negative associations, $0 \%$ positive associations, $0 \%$ non-linear associations, $57 \%$ show no association; Girls in MICs $(N=18) 6 \%$ negative associations, $78 \%$ positive associations, $0 \%$ non-linear associations, $17 \%$ show no association; Boys in MICs $(N=9) 11 \%$ negative associations, $63 \%$ positive associations, $0 \%$ non-linear associations, $26 \%$ show no association.

In both HICs and MICs, only five papers presented sex-specific analysis, with 15 and 31 associations reported, respectively. Positive associations were reported $86 \%$ of the time in boys, and $88 \%$ of the time in girls (Fig. 3). Results for sex-specific analysis in MICs was more similar to the pooled results, although there were slightly greater number of positive associations in boys (53\%) compared with girls (46\%).

In high quality papers $\left(\geq 7^{*}\right)$ the percentage of positive associations reported were lower than in the full analysis, with only three out of 12 associations (25\%), from seven papers, finding greater socioeconomic advantage related to higher FFM, and the rest reporting no association. Similar to the full analysis, HICs reported positive associations more frequently with three out of seven associations (43\%), from five papers, whilst in MICs all five associations coming from only two papers reported no overall pattern. In low quality papers $\left(\leq 3^{*}\right)$, the number of positive associations in HICs is lower compared to the full analysis with three of nine associations (33\%) from four papers reporting such a finding. In MICs, the seven positive associations out of 12 (58\%) were from just two papers, both using the same sample population.

\section{Childhood SEP and ratio and distribution measures}

Five papers reported on the association between SEP and a ratio or distribution measure (Table 5). Two papers used the same sample from the US and the remaining papers reported results from the UK $(n=2)$ and Brazil $(n=1)$.

Four papers looked at the association between SEP and a measure of central fat. Two of these using the same sample found greater social advantage to be associated with decreases in trunk FM, total abdominal adipose tissue [35] and trunk FMI [75]. The other two papers found no association between any SEP variable considered and central fat $[59,64]$. Only one paper looked at a ratio measure and found a lower mean muscle to fat ratio in lower parental income groups, except in girls aged 11-14 [61].

\section{DISCUSSION}

This systematic review finds evidence of socioeconomic inequalities in body composition in childhood and adolescence, although the direction and strength of these inequalities varies by measure of SEP, measure of body composition, sex and economic development of the country of study. Evidence of negative associations were generally observed for all measures of fat.
Negative associations were more frequently observed in samples from HICs compared with MICs, with positive associations only observed in studies from MICs. In HICs, negative associations were found more frequently in girls compared with boys, whilst in MICs girls more often showed positive associations. Greater socioeconomic advantage was associated with greater FFM in approximately half of the associations studied, but such associations were less common with outcome measures indexed to body size in both HICs and MICs, and in studies rated as higher quality. The review highlighted a lack of research using area-level measures of SEP, parental income and using more detailed measures of body composition, such as ratio and distribution measures.

Our findings for fat mass are broadly consistent with those from reviews using BMI as the measure of adiposity. Shrewsbury and Wardle [2] and Barriuso et al. [3], found associations between greater socioeconomic disadvantage and higher levels of adiposity among children and adolescents from HICs, identifying almost no associations in the opposite direction. Sobal and Stunkard [4] and Dinsa et al. [81] observed consistent evidence of more disadvantaged SEP being related to lower levels of obesity among children in MICs. However, where Sobal and Stunkard [4] found the association between SEP and obesity, measured largely by $\mathrm{BMI}$, to be inconsistent among children in HICs, we found more consistent evidence that greater socioeconomic disadvantage was associated with higher fat mass.

Previous research using anthropometric measures of obesity have reported that, in $\mathrm{HICs}$, studies using parental education report inverse associations most frequently $[2,3]$ consistent with our findings. Area-level measures of SEP have previously been shown to be particularly strong predictors of obesity [26], and this may be due to the close link between area-level measures of SEP and obesogenic elements in the environment $[82,83]$. However, we found very few studies using area-level SEP and body composition.

There are a greater percentage of negative associations between SEP and fat measures among children compared with those found in our review of adults in HICs [20]. This difference may indicate life course differences in the association between SEP and adiposity, or secular changes in inequalities given that the studies conducted in children typically include individuals born more recently than those conducted in adults. A comparison of the British birth cohorts demonstrated increasing inequalities in BMI with age within the cohorts, and inequalities in childhood and 
Table 4. Summary of associations between socioeconomic position and fat-free measures in children.

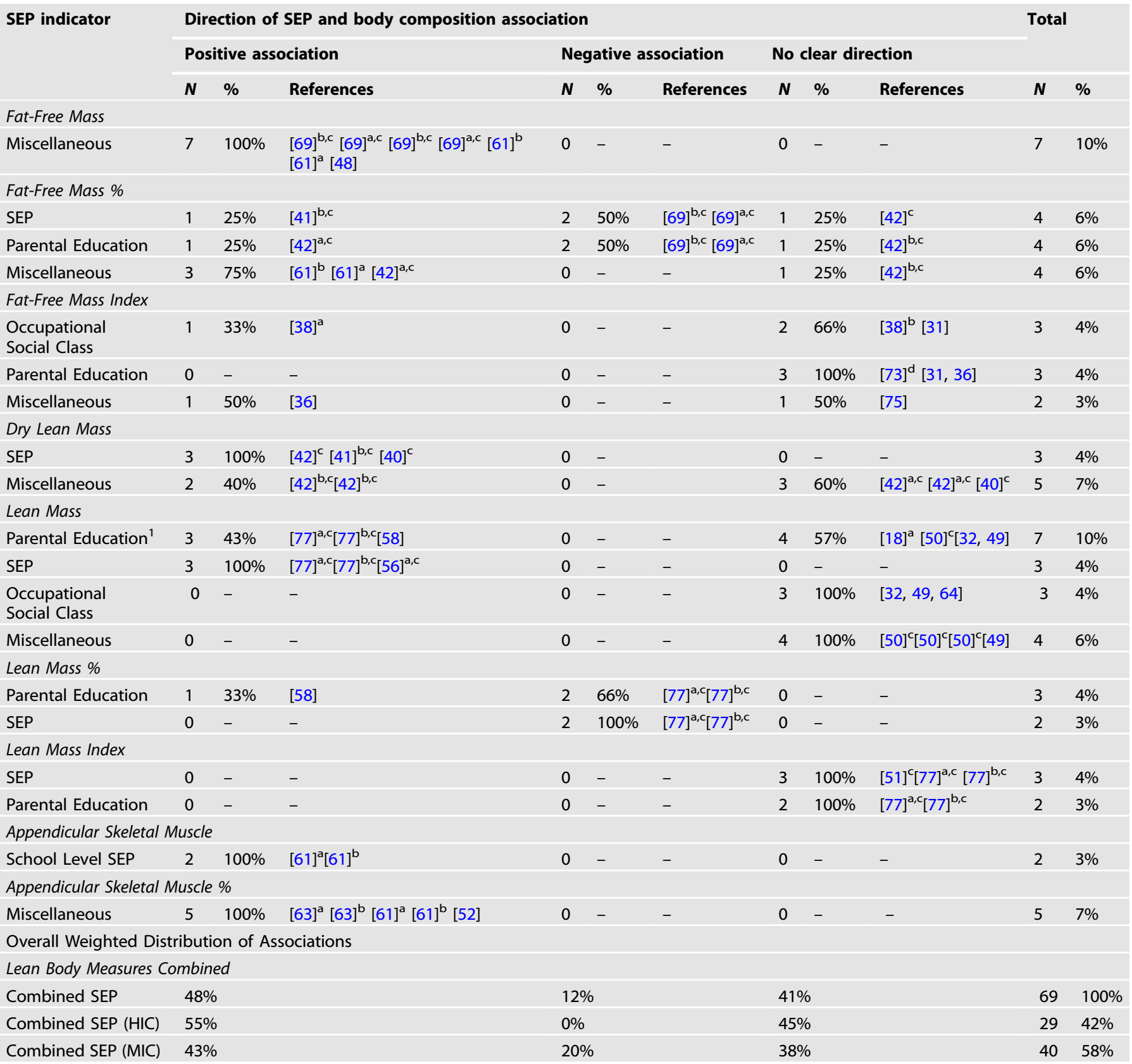

Positive associations indicate an increase in fat measure with an increase in socioeconomic advantage; inverse associations indicate a decrease in fat measure with an increase in socioeconomic advantage. Miscellaneous SEP measures are where less than two papers reported on the measure.

andicates results for girls only.

${ }^{b}$ Indicates results for boys only.

Indicates study conducted in a MIC.

Indicates lean body mass instead of FFM.

adolescents were only observed in the most recently born cohorts [84]. Research using the Fels Longitudinal Study demonstrated a secular increase in FM\% in children and adolescents from 1960 to 1999 [13]. Our results on directly measured adiposity in children compared to our results in adults [20] broadly match the trends seen in studies demonstrating secular increases in the inequalities in BMI [15, 84, 85]. Follow up of childhood cohorts into adulthood will be needed to distinguish a secular trend from an age effect.

Most studies in this review were conducted in children born post 1984, which means that in HICs they were all born into an obesogenic environment, the onset of which is generally estimated to be in the 1980s [86, 87]. Disadvantaged SEP, after the onset of the obesogenic environment, has been associated with increased proximity to fast food outlets [88, 89], larger advertising of fast food [90], and worse access to sports facilities [91] and green spaces [92] in HICs. Children are particularly influenced by advertising [93] and the food environment [94] and are less likely than adults to have a beneficial relationship between the built environment and levels of physical activity [95]. 


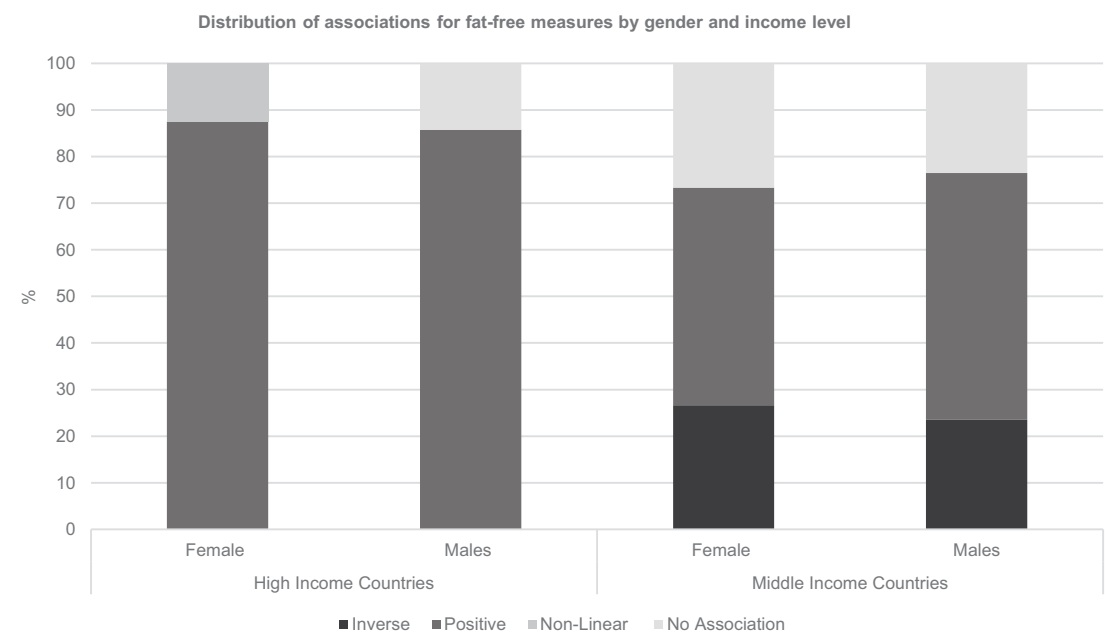

Fig. 3 Distribution of associations for fat-free measures by gender and income level. Girls in HICs $(N=8): 0 \%$ negative associations, $87.5 \%$ positive associations, $12.5 \%$ non-linear associations, $40 \%$ show no association; Boys in HICs ( $N=7$ ): $0 \%$ negative associations, $86 \%$ positive associations, $0 \%$ non-linear associations, $14 \%$ show no association; Girls in MICs $(N=15) 27 \%$ negative associations, $47 \%$ positive associations, $0 \%$ non-linear associations, $27 \%$ show no association; Boys in MICs $(N=17) 24 \%$ negative associations, $53 \%$ positive associations, $0 \%$ nonlinear associations, $24 \%$ show no association.

In contrast to HICs, disadvantaged SEP was associated with lower levels of body fat for children in MICs. Children of advantaged SEP in these countries have greater exposure to a western lifestyle compared to those of disadvantaged SEP, and in particular greater access to more expensive and energy dense foods [81]. Transnational food companies that have expanded to MICs often target children with their adverts, therefore making children particularly vulnerable to their efforts to increase purchase and consumption in MICs [96]. In addition, differences in physical activity may play a role. A study from India found higher rates of obesity in private schools compared with government schools [97], explained in part by a greater reliance on cars or buses to get to private schools, whilst children who attended government schools were more likely to walk or cycle [96]. There may also be cultural differences in perception of obesity between HICs and MICs [98], with overweight children in MICs being considered healthier by parents [97] and poorer understanding of the health consequences of obesity among mothers in MICs [99].

We previously reported on the association between SEP and fatfree measures in adulthood, finding predominantly no association, although with slight evidence of positive associations among women in HICs [20]. In contrast, we found considerable evidence for inequalities in fat-free measures in childhood, especially in HICs, and with few differences in associations between boys and girls.

Greater inequalities in FFM in childhood compared to adulthood may reflect a secular decline in levels of FFM, which are likely to be accompanied by growing inequalities, in the opposite direction to inequalities seen for adiposity. The Fels Longitudinal Study has shown mean FFMI to be lower in boys born in the 1990s compared with boys of the same age born decades before [13]. Serial data more recently has shown a secular decline in muscle strength, measured by handgrip, sit-ups, bent-arm hang and standing broad-jump tests, among children in the UK [14]. It is likely that secular changes in body composition would coincide with secular change in the inequalities, as has been observed with BMI [84], especially as changes to body composition have occurred alongside an overall increase in health inequalities [100]. Peak muscle function is determined across childhood and early adulthood and then maintained through midlife [101], and early development has been shown to be an important determinant of LM in later adult life [102]. It is therefore probable that inequalities in FFM observed in more recent generations in children are likely to persist into adulthood and old age.

In our review, few studies used indexed measures of FFM that aim to, at least partially, remove the correlation with height, nor did they adjust for height. Among those that did, there were fewer observed positive associations. Associations of greater disadvantage and lower FFM may therefore be explained, at least partially by height, as there is evidence that disadvantaged SEP is associated with shorter height across childhood and adulthood in most populations [103], although in HICs there is evidence this inequality has narrowed [84]. Positive associations in FFM in MICs may, however, also reflect that adequate nutrition is required for the development of muscle tissue as well as height, specifically intake of protein and micronutrients [104-108]. Increases in fat mass are accompanied by adaptive increases in lean mass $[17,109,110]$ and this may explain the association between disadvantaged SEP and lower fat-free measures in MICs, as children in disadvantaged SEP are more likely to be food insecure and lack essential macro and micronutrients [111], and therefore be shorter and have lower levels of both FM and FFM [112]. Further studies which appropriately adjust FFM measures for height are required to assess this. Few studies adjusted for fat mass, which is a suggested way of identifying the independent inequalities in lean mass.

\section{Strengths and limitations}

This review was registered with PROSPERO and has been carried out according to the published protocol [21]. The review has a generous inclusion criterion, capturing a broad range of evidence, thereby reducing selection bias. We also reduced bias by having two independent reviewers conducting each stage of the review, including selection of studies into the review and extraction of data, as well as completion of a quality assessment which was used to inform of the variability in study quality.

The generous inclusion criteria resulted in considerable heterogeneity in samples, study design and measures used. This variation, together with heterogeneity in the analytical approaches and reporting of results, prevented us from being able to conduct a meta-analysis. Additionally, the association, not paper, was used as the unit of analysis since most papers reported more than one association, meaning that in some cases one paper may contribute more weight to the overall summary of findings. The same data sets were also used by multiple authors in multiple papers. 


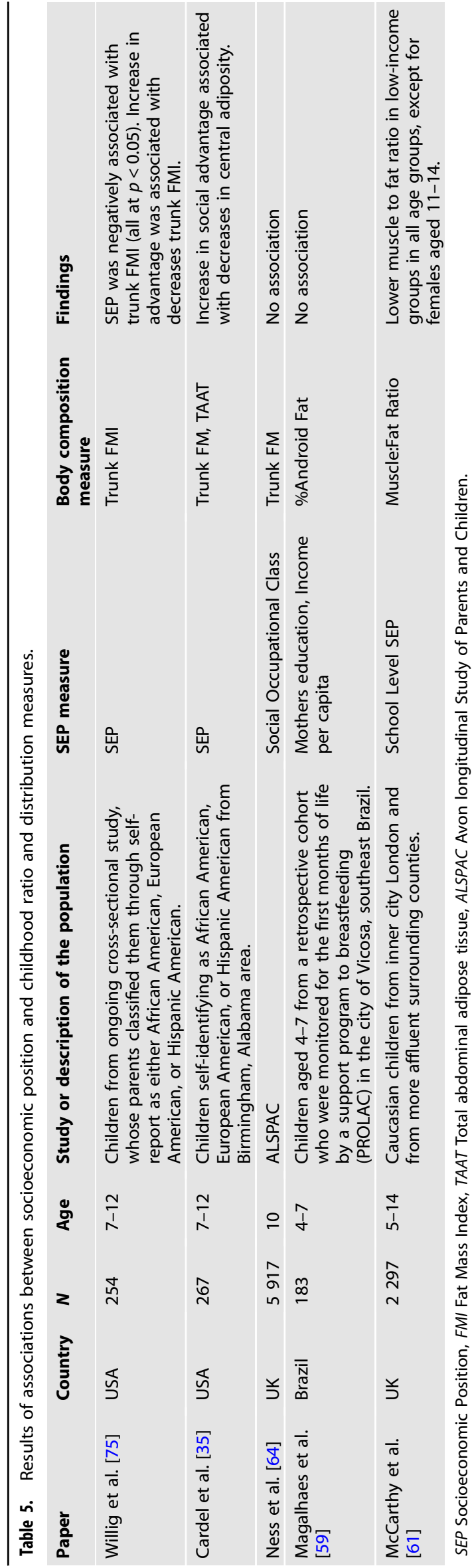

As it was not possible to conduct a meta-analysis, it was not possible to assess publication bias. It is, however, possible that publication bias exists with papers based on small sample sizes showing positive results are more likely to be published than those showing null findings. We did, however, include studies that tested the association of interest as part of a wider set of analyses instead of just focussing on those studies with a specific hypothesis on SEP differences, which may have reduced the impact of publication bias. This included papers that did not report full results where associations were found to be nonsignificant in preliminary analysis. However, this does mean that associations may not have appropriate adjustment for confounders.

This review prioritised assigning patterns to associations using the effect estimates and confidence intervals, which convey more about the direction and strength of effect, and the accuracy of these estimates $[113,114]$ and to overcome problems related to a reliance on $P$ values [114, 115]. However, many of the papers included reported $P$ values alongside only descriptive data or description of the association in the text. As $P$ values are influenced by the sample size of the study, lack of associations observed in such studies is likely due to a lack of statistical power. The studies included in this review were generally small, with a median sample size between 485 and 502. Additionally, because of the heterogeneity in SEP measures, outcomes, statistical approaches and the reporting of results it was not possible to make comparisons of effect size across papers, even among those studies that did use appropriate statistical methods. There may also have been overadjustment as studies have adjusted for factors which may actually be mediators rather than confounders.

There is inconsistency in the literature relating to the terminology used to describe FFM [116]. It is not uncommon for the same terminology to be used for different measures of FFM, or indeed different terminology to be used for a single measure. We sought to ensure comparability of results by applying standard definitions of FFM measures across the review, but some papers did not provide enough clarity on the measures used to do this confidently. There is a need for consistent definitions to be applied across the body composition literature, and for authors to provide clarity on the measures used.

We amended protocol slightly, due to the need to analyse heterogeneity by country income level, as more papers were identified from MICs than expected and it was clear that this was an unignorable source of heterogeneity. We had no papers included from LICs, limiting the ability to explore SEP and body composition associations in countries at an earlier stage of the nutrition transition, which would have been valuable for understanding the changing relationship of SEP and obesity with economic development.

\section{Implications}

In monitoring inequalities, BMI may accurately capture fat mass at a population level in childhood, given the similarities of our findings with reviews on social inequalities in BMI. However, our review suggests contrasting findings on inequalities in FFM in HICs. This may, assuming such associations are not fully explained by inequalities in height, mean that BMI underestimates the inequalities in the health risks related to adiposity. If children from more disadvantaged SEP groups have lower levels of muscle mass and strength, as well as higher levels of fat mass, this may have important implications for inequalities in outcomes which also require good muscle function. As we have found greater evidence of inequalities in body composition in children compared with adults, tracking of body composition through the life course in more recent generations could have important implications for inequalities in physical capability in later life. Follow up of these childhood cohorts is needed to confirm whether these are secular rather than age-related changes in inequalities. 
It is also crucial that future research appropriately indexes body composition measures and distinguishes measures that include bone from those that do not. Inequalities in height are likely to be an important factor that can explain the observed inequalities in such measures. The results of this review also highlight the need for more research investigating the associations of SEP with ratio and distribution measures of body composition which are related to metabolic and cardiovascular disease outcomes, especially in MICs, and greater research on sex differences in both HICs and MICs. There are also gaps in research looking at the effect of arealevel measures on body composition, a measure of SEP that is closely linked to the obesogenic environment, particularly in relation to FFM.

Efforts should be made to address inequalities in both FM and FFM among children in HICs and MICs by reducing access to, and advertising of, fast food to children, and promoting and ensuring equal access to healthy and nutritious food. Promotion of physical activity and access to sport facilities should also be prioritised in poorer communities to address inequalities in FFM.

\section{REFERENCES}

1. Simmonds M, Llewellyn A, Owen CG, Woolacott N. Predicting adult obesity from childhood obesity: a systematic review and meta-analysis. Obes Rev. 2016;17:95-107.

2. Shrewsbury V, Wardle J. Socioeconomic status and adiposity in childhood: a systematic review of cross-sectional studies 1990-2005. Obesity. 2008;16:275-84.

3. Barriuso L, Miqueleiz E, Albaladejo R, Villanueva R, Santos JM, Regidor E. Socioeconomic position and childhood-adolescent weight status in rich countries: a systematic review, 1990-2013. BMC Pediatr. 2015;15:129.

4. Sobal J, Stunkard AJ. Socioeconomic status and obesity: a review of the literature. Psychol Bull. 1989;105:260-75.

5. Wells JC, Fewtrell MS. Measuring body composition. Arch Dis Child. 2006;91:612-7.

6. Srikanthan $\mathrm{P}$, Horwich $\mathrm{TB}$, Tseng $\mathrm{CH}$. Relation of muscle mass and fat mass to cardiovascular disease mortality. Am J Cardiol. 2016;117:1355-60.

7. Kang SM, Yoon JW, Ahn HY, Kim SY, Lee KH, Shin H, et al. Android fat depot is more closely associated with metabolic syndrome than abdominal visceral fat in elderly people. PLoS One. 2011;6:11

8. Samsell L, Regier M, Walton C, Cottrell L. Importance of android/gynoid fat ratio in predicting metabolic and cardiovascular disease risk in normal weight as well as overweight and obese children. J Obes. 2014;2014:846578.

9. Vasan SK, Osmond C, Canoy D, Christodoulides C, Neville MJ, Di Gravio C, et al. Comparison of regional fat measurements by dual-energy $\mathrm{X}$-ray absorptiometry and conventional anthropometry and their association with markers of diabetes and cardiovascular disease risk. Int J Obes. 2018;42:850-7.

10. Coutinho T, Goel K, Corrêa de Sá D, Kragelund C, Kanaya AM, Zeller M, et al. Central obesity and survival in subjects with coronary artery disease: a systematic review of the literature and collaborative analysis with individual subject data. J Am Coll Cardiol. 2011;57:1877-86.

11. Takamura T, Kita $Y$, Nakagen $M$, Sakurai $M$, Isobe $Y$, Takeshita $Y$, et al. Weightadjusted lean body mass and calf circumference are protective against obesityassociated insulin resistance and metabolic abnormalities. Heliyon. 2017;3: e00347.

12. Yliharsila H, Kajantie E, Osmond C, Forsen T, Barker DJ, Eriksson JG. Body mass index during childhood and adult body composition in men and women aged 56-70 y. Am J Clin Nutr. 2008;87:1769-75.

13. Sun SS, Deng X, Sabo R, Carrico R, Schubert CM, Wan W, et al. Secular trends in body composition for children and young adults: the Fels Longitudinal Study. Am J Hum Biol. 2012;24:506-14.

14. Sandercock GRH, Cohen DD. Temporal trends in muscular fitness of English 10year-olds 1998-2014: an allometric approach. J Sci Med Sport. 2018;22:201-5.

15. Bann D, Johnson W, Li L, Kuh D, Hardy R. Socioeconomic inequalities in body mass index across adulthood: coordinated analyses of individual participant data from three british birth cohort studies initiated in 1946, 1958 and 1970. PLoS Med. 2017;14:e1002214.

16. Goisis A, Sacker A, Kelly Y. Why are poorer children at higher risk of obesity and overweight? A UK cohort study. Eur J Public Health. 2016;26:7-13.

17. Cooper R, Hardy R, Bann D, Aihie Sayer A, Ward KA, Adams JE, et al. Body mass index from age 15 years onwards and muscle mass, strength, and quality in early old age: findings from the mrc national survey of health and development. J Gerontol A Biol Sci Med Sci. 2014;69:1253-9.
18. Cheng S, Völgyi E, Tylavsky FA, Lyytikäinen A, Törmäkangas $T$, Xu L, et al. Traitspecific tracking and determinants of body composition: a 7-year follow-up study of pubertal growth in girls. BMC Med. 2009;7:5.

19. Guo SS, Chumlea WC, Roche AF, Siervogel RM. Age- and maturity-related changes in body composition during adolescence into adulthood: the Fels longitudinal study. Appl Radiat Isot. 1998;49:581-5.

20. Bridger Staatz C, Kelly Y, Lacey R, Arnot M, Blodgett J, George A, et al. Life course socioeconomic position and body composition in adulthood: A systematic review and narrative synthesis. Int J Obes.

21. Bridger Staatz C, Kelly $Y$, Lacey R, Hardy R. Socioeconomic position and body composition across the life course: a systematic review protocol. Syst Rev. 2019;8:263.

22. Ouzzani M, Hammady H, Fedorowicz Z, Elmagarmid A. Rayyan-a web and mobile app for systematic reviews. Syst Rev. 2016;5:210.

23. Wells G, Shea B, O'Connell D, Peterson J, Welch V, Losos M, et al. The NewcastleOttawa Scale (NOS) forassessing the quality of nonrandomised studies in metaanalyses. 2013. http://www.ohri.ca/programs/clinical_epidemiology/oxford.asp.

24. Rohatgi A. WebPlotDigitizer Pacifica, California, USA. 2020. https://automeris.io/ WebPlotDigitizer.

25. Popay JR, Sowden H, Petticrew A, Arai M, Rodgers L, Britten M, et al. Guidance on the conduct of narrative synthesis in systematic reviews. Lancaster: Lancaster University; 2006.

26. McLaren L. Socioeconomic status and obesity. Epidemiol Rev. 2007;29:29-48.

27. Ball K, Crawford D. Socioeconomic status and weight change in adults: a review. Soc Sci Med. 2005;60:1987-2010.

28. The World Bank. World Bank Country and Lending Groups: The World Bank; 2019. https://datahelpdesk.worldbank.org/knowledgebase/articles/906519world-bank-country-and-lending-groups.

29. Apouey BH. Child physical development in the UK: the imprint of time and socioeconomic status. Public Health. 2016;141:255-63.

30. Azcorra H, Dickinson F, Datta, Banik S. Maternal height and its relationship to offspring birth weight and adiposity in 6- to 10-year-old Maya children from poor neighborhoods in Merida, Yucatan. Am J Phys Anthropol. 2016;161:571-9.

31. Baird J, Hill CM, Harvey NC, Crozier S, Robinson SM, Godfrey KM, et al. Duration of sleep at 3 years of age is associated with fat and fat-free mass at 4 years of age: the Southampton Women's Survey. J Sleep Res. 2016;25:412-8.

32. Boot AM, Bouquet J, de Ridder MA, Krenning EP, de Muinck, Keizer-Schrama SM. Determinants of body composition measured by dual-energy X-ray absorptiometry in Dutch children and adolescents. Am J Clin Nutr. 1997;66:232-8.

33. Brown DE, Gotshalk LA, Katzmarzyk PT, Allen L. Measures of adiposity in two cohorts of Hawaiian school children. Ann Hum Biol. 2011;38:492-9.

34. Burdette HL, Whitaker RC, Hall WC, Daniels SR. Breastfeeding, introduction of complementary foods, and adiposity at $5 \mathrm{y}$ of age. Am J Clin Nutr. 2006;83:550-8.

35. Cardel M, Willig AL, Dulin-Keita A, Casazza K, Beasley TM, José RF. Parental feeding practices and socioeconomic status are associated with child adiposity in a multi-ethnic sample of children. Appetite. 2012;58:347-53.

36. Carter PJ, Taylor BJ, Williams SM, Taylor RW. Longitudinal analysis of sleep in relation to BMI and body fat in children: the FLAME study. BMJ. 2011;342: d2712.

37. Castro NP, Euclydes VV, Simões FA, Vaz-de-Lima LR, De Brito CA, Luzia LA, et al. The relationship between maternal plasma leptin and adiponectin concentrations and newborn adiposity. Nutrients. 2017;9:23.

38. Chomtho S, Wells JC, Williams JE, Lucas A, Fewtrell MS. Associations between birth weight and later body composition: evidence from the 4-component model. Am J Clin Nutr. 2008;88:1040-8.

39. Collings PJ, Wijndaele K, Corder K, Westgate K, Ridgway CL, Sharp SJ, et al. Objectively measured physical activity and longitudinal changes in adolescent body fatness: an observational cohort study. Pediatr Obes. 2015;11:107-14.

40. Datta Banik S, Castillo T, Rodriguez L, Dickinson F. Body fatness in relation to physical activity and selected socioeconomic parameters of adolescents aged 15-17 years in Merida, Yucatan. Ann Hum Biol. 2014;41:497-505.

41. Datta Banik S, Andrade A, Dickinson F. Adiposity and body composition of 13and 14-year-old boys in Merida, Mexico: A study in relation with macronutrient consumption rates and socioeconomic status. Obesity. 2011;1:S214-S5.

42. Datta Banik S, Andrade Olalde AC, Rodriguez L, Dickinson F. The effect of socioeconomic indicators and macronutrient intake rate on body composition in adolescents 12 to 16 years old in Merida, Yucatan. Anthropol Anz. 2014;71:347-68.

43. De Vriendt T, Clays E, Maes L, De Bourdeaudhuij I, Vicente-Rodriguez G, Moreno LA, et al. European adolescents' level of perceived stress and its relationship with body adiposity-the HELENA Study. Eur J Public Health. 2011;22:519-24.

44. Dowda M, Taverno Ross SE, Mclver KL, Dishman RK, Pate RR. Physical activity and changes in adiposity in the transition from elementary to middle school. Child Obes. 2017;13:53-62. 
45. Duncan JS, Schofield G, Duncan EK, Rush EC. Risk factors for excess body fatness in New Zealand children. Asia Pac J Clin Nutr. 2008;17:138-47.

46. Ebenegger V, Marques-Vidal PM, Nydegger A, Laimbacher J, Niederer I, Bürgi F, et al. Independent contribution of parental migrant status and educational level to adiposity and eating habits in preschool children. Eur J Clin Nutr. 2011;65:210-8.

47. Ekelund U, Neovius M, Linne Y, Brage S, Wareham NJ, Rossner S. Associations between physical activity and fat mass in adolescents: the Stockholm Weight Development Study. Am J Clin Nutr. 2005;81:355-60.

48. Ekelund U, Ong K, Linné $Y$, Neovius $M$, Brage $S$, Dunger DB, et al. Upward weight percentile crossing in infancy and early childhood independently predicts fat mass in young adults: the Stockholm Weight Development Study (SWEDES). Am J Clin Nutr. 2006;83:324-30.

49. Gracia-Marco L, Ortega FB, Casajús JA, Sioen I, Widhalm K, Béghin L, et al. Socioeconomic status and bone mass in Spanish adolescents. The HELENA Study. J Adolesc Health. 2012;50:484-90.

50. Griffiths PL, Johnson W, Cameron N, Pettifor JM, Norris SA. In urban South Africa, 16 year old adolescents experience greater health equality than children. Econ Hum Biol. 2013;11:502-14.

51. Griffiths PL, Rousham EK, Norris SA, Pettifor JM, Cameron N. Socio-economic status and body composition outcomes in urban South African children. Arch Dis Child. 2008;93:862-7.

52. Hou WW, Lam TH, Leung GM, Schooling CM. Adolescent testosterone, muscle mass and glucose metabolism: Evidence from "children of 1997" in hong kong. Am J Epidemiol. 2014;11:S93.

53. Howe LD, Galobardes B, Sattar N, Hingorani AD, Deanfield J, Ness AR, et al. Are there socioeconomic inequalities in cardiovascular risk factors in childhood, and are they mediated by adiposity? Findings from a prospective cohort study. Int J Obes. 2010;34:1149-59.

54. Howe LD, Lawlor DA, Propper C. Trajectories of socioeconomic inequalities in health, behaviours and academic achievement across childhood and adolescence. J Epidemiol Community Health. 2013;67:358-64.

55. Johnson LM, Er AP, Jones LR, Emmett PM, Jebb SA. A prospective analysis of dietary energy density at age 5 and 7 years and fatness at 9 years among UK children. Int J Obes. 2008;32:586-93.

56. Khadilkar AV, Sanwalka NJ, Kadam NS, Chiplonkar SA, Khadilkar VV, Mughal MZ. Poor bone health in underprivileged Indian girls: an effect of low bone mass accrual during puberty. Bone. 2012;50:1048-53.

57. Lagoa MJ, Silva G, Alves Al, Medeiros AF, Mota J, Aires L. Parent-children resemblance and associations of family behaviour on children body fat. Obesity Facts. 2015;1:120.

58. Lantz H, Bratteby LE, Fors H, Sandhagan B, Sjöström L, Samuelson G. Body composition in a cohort of Swedish adolescents aged 15, 17 and 20.5 years. Acta Paediatr. 2008;97:1691-7.

59. Magalhães TC, Vieira SA, Priore SE, Ribeiro AQ, Lamounier JA, Franceschini SC, et al. Exclusive breastfeeding and other foods in the first six months of life: effects on nutritional status and body composition of Brazilian children. Thescientificworldjournal. 2012;2012:468581.

60. Matsudo VK, Ferrari GL, Araújo TL, Oliveira LC, Mire E, Barreira TV, et al. Socioeconomic status indicators, physical activity, and overweight/obesity in Brazilian children. Revista Paulista de Pediatria. 2016;34:162-70.

61. McCarthy HD, Van Eeden C, Samani-Radia D. Influence of socioeconomic status on measures of skeletal muscle mass in children. Proceedings of the Nutrition Society. 2015;74(OCE2):E153.

62. Molina-Garcia J, Queralt A, Adams MA, Conway TL, Sallis JF. Neighborhood built environment and socio-economic status in relation to multiple health outcomes in adolescents. Prev Med. 2017;105:88-94.

63. Moon JH, Kong MH, Kim HJ. Low muscle mass and depressed mood in Korean adolescents: a cross-sectional analysis of the fourth and fifth Korea National Health and Nutrition Examination Surveys. J Korean Med Sci. 2018;33:e320.

64. Ness AR, Leary S, Reilly J, Wells J, Tobias J, Clark E, et al. The social patterning of fat and lean mass in a contemporary cohort of children. Int J Pediatr Obes. 2006;1:59-61.

65. Nguyen TT, Butte NF, Wang Y. Demographic and socioeconomic correlates of adiposity assessed with dual-energy X-ray absorptiometry in US children and adolescents. Am J Clin Nutr. 2012;96:1104-12.

66. Nguyen TT, Butte NF, Wang Y. Demographic and socioeconomic correlates of body fat assessed using DXA in US children and adolescents. FASEB J Conf Exp Biol. 2011;25:974.14-974.14.

67. Plachta-Danielzik S, Kehden B, Starke F, Muller MJ. Family and lifestyle factors mediate the association between socio-economic status and fat mass in children and adolescents. Obesity Facts. 2015;1:145.

68. Samani-Radia D, McCarthy HD. Comparison of children's body fatness between two contrasting income groups: contribution of height difference. Int J Obes. 2011;35:128-33.
69. Santos IS, Barros AJ, Matijasevich A, Zanini R, Chrestani Cesar MA, et al. Cohort profile update: 2004 pelotas (Brazil) birth cohort study. Body composition, mental health and genetic assessment at the 6 years follow-up. Int J Epidemiol. 2014;43:1437-1437a-f.

70. Schaefer SE, Salazar M, Bruhn C, Saviano D, Boushey C, Van, et al. Influence of race, acculturation, and socioeconomic status on tendency toward overweight in Asian-American and Mexican-American early adolescent females. J Immigr Minor Health. 2009;11:188-97.

71. Shakir RN, Coates AM, Olds T, Rowlands A, Tsiros MD. Not all sedentary behaviour is equal: children's adiposity and sedentary behaviour volumes, patterns and types. Obesity Res Clin Pract. 2018;12:506-12.

72. Ulbricht L, de Campos MF, Esmanhoto E, Ripka WL. Prevalence of excessive body fat among adolescents of a south Brazilian metropolitan region and State capital, associated risk factors, and consequences. BMC Public Health. 2018;18:312.

73. van den Berg G, van Eijsden M, Vrijkotte TG, Gemke RJ. BMI may underestimate the socioeconomic gradient in true obesity. Pediatr Obes. 2012;8:e37-40.

74. Veena SR, Hegde BG, Ramachandraiah S, Krishnaveni GV, Fall CH, Sirinivasan K. Relationship between adiposity and cognitive performance in 9-10-year-old children in South India. Arch Dis Child. 2014;99:126-34.

75. Willig AL, Hanks LJ, Fernandez JR. Birth weight is associated with body composition in a multiethnic pediatric cohort. Open Obes J.2011;3:4-8.

76. Wohlfahrt-Veje C, Tinggaard J, Winther K, Mouritsen A, Hagen CP, Mieritz MG, et al. Body fat throughout childhood in 2647 healthy Danish children: agreement of BMI, waist circumference, skinfolds with dual X-ray absorptiometry. Eur J Clin Nutr. 2014;68:664-70.

77. Zanini RV, Santos IS, Gigante DP, Matijasevich A, Barros FC, Barros AJ. Body composition assessment using DXA in six-year-old children: the 2004 Pelotas Birth Cohort, Rio Grande do Sul State, Brazil. Cad Saude Publica. 2014;30:2123-33.

78. Janmahasatian S, Duffull SB, Ash S, Ward LC, Byrne NM, Green B. Quantification of lean bodyweight. Clin Pharmacokinet. 2005;44:1051-65.

79. Buckinx F, Landi F, Cesari M, Fielding RA, Visser M, Engelke $K$, et al. Pitfalls in the measurement of muscle mass: a need for a reference standard. J Cachexia Sarcopenia Muscle. 2018;9:269-78.

80. Hansen RD, Raja C, Aslani A, Smith RC, Allen BJ. Determination of skeletal muscle and fat-free mass by nuclear and dual-energy $x$-ray absorptiometry methods in men and women aged 51-84 y (1-3). Am J Clin Nutr. 1999;70:228-33.

81. Dinsa GD, Goryakin Y, Fumagalli E, Suhrcke M. Obesity and socioeconomic status in developing countries: a systematic review. Obes Rev. 2012;13:1067-79.

82. Navalpotro L, Regidor E, Ortega P, Martinez D, Villanueva R, Astasio P. Areabased socioeconomic environment, obesity risk behaviours, area facilities and childhood overweight and obesity: socioeconomic environment and childhood overweight. Prev Med. 2012;55:102-7.

83. Tyrrell J, Wood AR, Ames RM, Yaghootkar H, Beaumont RN, Jones SE, et al. Geneobesogenic environment interactions in the UK Biobank study. Int J Epidemiol. 2017;46:559-75.

84. Bann D, Johnson W, Li L, Kuh D, Hardy R. Socioeconomic inequalities in childhood and adolescent body-mass index, weight, and height from 1953 to 2015: an analysis of four longitudinal, observational, British birth cohort studies. Lancet Public Health. 2018;3:E194-203.

85. Senese LC, Almeida ND, Fath AK, Smith BT, Loucks EB. Associations between childhood socioeconomic position and adulthood obesity. Epidemiol Rev. 2009;31:21-51.

86. Cohen DA. Obesity and the built environment: changes in environmental cues cause energy imbalances. Int J Obes. 2008;32:S137-42.

87. Maguire ER, Burgoine T, Monsivais P. Area deprivation and the food environment over time: a repeated cross-sectional study on takeaway outlet density and supermarket presence in Norfolk, UK, 1990-2008. Health Place. 2015;33:142-7.

88. Cetateanu A, Jones A. Understanding the relationship between food environments, deprivation and childhood overweight and obesity: Evidence from a cross sectional England-wide study. Health \& Place. 2014;27:68-76.

89. Hurvitz PM, Moudon AV, Rehm CD, Streichert LC, Drewnowski A. Arterial roads and area socioeconomic status are predictors of fast food restaurant density in King County. WA. Int J Behav Nutr Phy. 2009;6:6.

90. Adams J, Ganiti E, White M. Socio-economic differences in outdoor food advertising in a city in Northern England. Public Health Nutr. 2011;14:945-50.

91. Gordon-Larsen P, Nelson MC, Page P, Popkin BM. Inequality in the built environment underlies key health disparities in physical activity and obesity. Pediatrics. 2006;117:417-24.

92. Hoffimann E, Barros H, Ribeiro Al. Socioeconomic inequalities in green space quality and accessibility-evidence from a southern European city. Int J Environ Res Public Health. 2017;14:8. 
93. Russell SJ, Croker H, Viner RM. The effect of screen advertising on children's dietary intake: a systematic review and meta-analysis. Obes Rev. 2019;20:554-68.

94. Osei-Assibey G, Dick S, Macdiarmid J, Semple S, Reilly JJ, Ellaway A, et al. The influence of the food environment on overweight and obesity in young children: a systematic review. Bmj Open. 2012;2:6

95. Ferdinand AO, Sen B, Rahurkar S, Engler S, Menachemi N. The relationship between built environments and physical activity: a systematic review. Am J Public Health. 2012;102:E7-E13.

96. Gupta N, Goel K, Shah P, Misra A. Childhood obesity in developing countries: epidemiology, determinants, and prevention. Endocr Rev. 2012;33:48-70.

97. Bhardwaj S, Misra A, Khurana L, Gulati S, Shah P, Vikram NK. Childhood obesity in Asian Indians: a burgeoning cause of insulin resistance, diabetes and subclinical inflammation. Asia Pac J Clin Nutr. 2008;17:172-5.

98. Rguibi M, Belahsen R. Body size preferences and sociocultural influences on attitudes towards obesity among Moroccan Sahraoui women. Body Image. 2006;3:395-400.

99. Hossain MS, Siddiqee MH, Ferdous S, Faruki M, Jahan R, Shahik SM, et al. Is childhood overweight/obesity perceived as a health problem by mothers of preschool aged children in Bangladesh? A community level cross-sectional study. Int J Environ Res Public Health. 2019;16:2.

100. Bartley M. Health inequality: an introduction to concepts, theories and methods. 2nd ed. Cambridge: Cambridge Polity Press; 2017.

101. Dodds RM, Syddall HE, Cooper R, Benzeval M, Deary IJ, Dennison EM, et al. Grip strength across the life course: normative data from twelve British studies. PLoS One. 2014;9:e113637.

102. Kuh D, Bassey J, Hardy R, Aihie Sayer A, Wadsworth M, Cooper C. Birth weight, childhood size, and muscle strength in adult life: evidence from a birth cohort study. Am J Epidemiol. 2002;156:627-33.

103. Peñuelas J, Janssens IA, Ciais $P$, Obersteiner $M$, Krisztin T, Piao $S$, et al. Increasing gap in human height between rich and poor countries associated to their different intakes of $\mathrm{N}$ and P. Sci Rep. 2017;7:17671.

104. Churchward-Venne TA, Burd NA, Phillips SM. Nutritional regulation of muscle protein synthesis with resistance exercise: strategies to enhance anabolism. Nutr Metab. 2012;9:40.

105. Phillips SM. A brief review of critical processes in exercise-induced muscular hypertrophy. Sports Med. 2014;44:S71-7.

106. Kulkarni B. Addressing the double burden of malnutrition in developing countries: need for strategies to improve the lean body mass. Food Nutr Bull. 2018;39:S69-S76.

107. Braun KV, Erler NS, Kiefte-de Jong JC, Jaddoe VW, van den Hooven EH, Franco $\mathrm{OH}$, et al. Dietary intake of protein in early childhood is associated with growth trajectories between 1 and 9 years of age. J Nutr. 2016;146:2361-7.

108. Perkins JM, Subramanian SV, Davey Smith G, Ozaltin E. Adult height, nutrition, and population health. Nutr Rev. 2016;74:149-65.

109. Chaston TB, Dixon JB, O'Brien PE. Changes in fat-free mass during significant weight loss: a systematic review. Int J Obes. 2007;31:743-50.

110. Forbes GB. Some adventures in body composition, with special reference to nutrition. Acta Diabetol. 2003;40:S238-S41.

111. Muller $\mathrm{O}$, Krawinkel M. Malnutrition and health in developing countries. CMAJ. 2005;173:279-86.

112. Wells JCK. Body composition of children with moderate and severe undernutrition and after treatment: a narrative review. BMC Med. 2019;17:215.
113. Wasserstein RL, Schirm AL, Lazar NA. Moving to a World Beyond "p $<0.05$ ". Am Stat. 2019;73:1-19.

114. Halsey LG. The reign of the p-value is over: what alternative analyses could we employ to fill the power vacuum? Biol Lett. 2019;15:5.

115. Sterne JA, Davey Smith G. Sifting the evidence-what's wrong with significance tests? BMJ. 2001;322:226-31.

116. Scafoglieri A, Clarys JP. Dual energy X-ray absorptiometry: gold standard for muscle mass? J Cachexia Sarcopenia Muscle. 2018;9:786-7.

\section{ACKNOWLEDGEMENTS}

CBS is supported by a PhD studentship from the UK Medical Research Council (MR/ N013867/1). RH is Director of the CLOSER consortium, which is supported by the Economic and Social Research Council (ESRC) (award reference: ES/K000357/1). YK and $\mathrm{RL}$ are supported by the ESRC (ES/R008930/1, ES/P010229/1). AG, MA and EW are supported by PhD Studentships from the ESRC (ES/P000347/1). JB was supported by the Canadian Institutes of Health Research (FDSA) and the Canadian Centennial Scholarship Fund.

\section{COMPETING INTERESTS}

The authors declare no competing interests.

\section{ADDITIONAL INFORMATION}

Supplementary information The online version contains supplementary material available at https://doi.org/10.1038/s41366-021-00899-y.

Correspondence and requests for materials should be addressed to C.B.S.

Reprints and permission information is available at http://www.nature.com/ reprints

Publisher's note Springer Nature remains neutral with regard to jurisdictional claims in published maps and institutional affiliations.

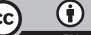

Open Access This article is licensed under a Creative Commons Attribution 4.0 International License, which permits use, sharing, adaptation, distribution and reproduction in any medium or format, as long as you give appropriate credit to the original author(s) and the source, provide a link to the Creative Commons license, and indicate if changes were made. The images or other third party material in this article are included in the article's Creative Commons license, unless indicated otherwise in a credit line to the material. If material is not included in the article's Creative Commons license and your intended use is not permitted by statutory regulation or exceeds the permitted use, you will need to obtain permission directly from the copyright holder. To view a copy of this license, visit http://creativecommons. org/licenses/by/4.0/

(c) The Author(s) 2021 